A\&A 639, A102 (2020)

https://doi.org/10.1051/0004-6361/202037989

(c) C. Salas-Matamoros and K.-L. Klein 2020

\title{
Polarisation and source structure of solar stationary type IV radio bursts ${ }^{\star}$
}

\author{
Carolina Salas-Matamoros ${ }^{1}$ and Karl-Ludwig Klein ${ }^{2}$
}

\author{
1 Space Research Center, University of Costa Rica, San Jose, Costa Rica \\ 2 Observatoire de Paris, LESIA \& Station de Radioastronomie de Nançay, Univ. PSL, CNRS, Sorbonne Univ., Univ. de Paris, \\ Univ. d'Orléans, 5 Place Jules Janssen, 92190 Meudon, France \\ e-mail: Ludwig.Klein@obspm.fr
}

Received 20 March 2020 / Accepted 16 May 2020

\begin{abstract}
The reconfiguration of the magnetic field during and after a coronal mass ejection (CME) may be accompanied by radio emission from non-thermal electrons. In particular, stationary type IV bursts (also called storm continua) are emitted by electrons in closed magnetic configurations usually located in the wake of the outward-travelling CME. Although stationary type IV bursts, which stand out by their long duration (up to several hours) and strong circular polarisation, have been known for more than fifty years, there have been no systematic studies since the 1980s. In this work we use the data pool of the Nançay Radioheliograph together with white-light coronagraphy, EUV imaging and magnetography from the SoHO, Proba2, SDO and STEREO spacecraft to revisit the source structure and polarisation of a sample of seven well-defined stationary type IV bursts at decimetre-to-metre wavelengths. The radio sources are most often found in one leg, in one case both legs, of the magnetic flux rope erupting into the high corona during the CME. The cross-correlation of the brightness temperature time profiles in the event with sources in both legs implies that the radiating electrons have energies of a few tens of keV. Comparison with the magnetic field measured in the photosphere and its potential extrapolation into the corona shows that the radio emission is in the ordinary mode. This result was inferred historically by means of the hypothesis that the magnetic field orientation in the radio source was that of the dominant sunspot in the parent active region. This hypothesis is shown here to be in conflict with noise storms in the same active region. It is confirmed that the polarisation of stationary type IV continua may be strong, but is rarely total, and that it gradually increases in the early phase of the radio event. We find that the increase is related to the gradual disappearance of some weakly polarised or unpolarised substructure, which dominates the first minutes of the radio emission.
\end{abstract}

Key words. acceleration of particles - Sun: coronal mass ejections (CMEs) - Sun: flares - Sun: magnetic fields Sun: radio radiation - solar-terrestrial relations

\section{Introduction}

A coronal mass ejection (CME) is generally understood to comprise a magnetic flux rope, which at some time starts to rise into the high corona (e.g. Démoulin \& Aulanier 2010; Howard et al. 2017; Song et al. 2019). It leaves behind a stressed magnetic field that will re-arrange itself over several hours. This configuration is the seat of time-extended energy release and a prime example of magnetic reconnection in the solar corona, leading for instance to the formation of arcades of flare loops (also called "post-flare loops"; Webb \& Howard 2012; Janvier et al. 2015). The wake of a CME is also of potential interest to particle acceleration. Prolonged signatures of non-thermal electrons are observed as broadband emission at radio wavelengths, which may extend from centimetric to decametric wavelengths. This emission has a variety of properties and is collectively referred to as type IV bursts.

Type IV bursts were initially (Boischot 1957) identified as a distinct category of metre wave radio emissions through interferometric observations, which showed outward-moving sources of broadband radio emission in relationship with some flares. It was then shown that similar broadband radio emissions exist

* The movie is available at https://www . aanda.org without systematic source motions. This led to the introduction of an increasing number of sub-categories of type IV bursts in the course of time. Their common aspects, besides being broadband, standing out at decimetric to decametric waves, are their association with flares and their duration that exceeds the impulsive flare phase. The association with flares distinguishes type IV bursts from noise storms, which are the most common signature of non-thermal electrons at radio wavelengths, accompanying quiescent active regions (e.g. Elgarøy 1977; Kai et al. 1985; Raulin \& Klein 1994; Bentley et al. 2000; Del Zanna et al. 2011). The long duration and broad instantaneous bandwidth distinguish type IV bursts from radio bursts of type III and type II. The moving type IV burst category, as it was then called, draws attention to the fact that type IV bursts as a category are associated not only with flares, but with largescale mass motions, such as filament eruptions (Riddle 1970; MacQueen 1980; Klein \& Mouradian 2002) and coronal mass ejections (CMEs) in general (see the reviews of early work on this subject by MacQueen 1980; Robinson 1986). Historical overviews on the development and ramification of the type IV burst concept can be found in Sect. 15.1 of Robinson (1985), in Pick (1986), and in Sect. 2.1.2 of Pick \& Vilmer (2008).

In her systematic study of type IV bursts Pick-Gutmann (1961) distinguished a first phase, from centimetric to metric 
wavelengths, which comprised the moving type IV burst, from a much longer lasting second phase of emission (up to several hours or even days) at decimetric-to-decametric waves, with significant circular polarisation. She called the second phase of emission storm continuum ("orage-continuum" in the French text). This emission was later termed stationary type IV burst (Weiss 1963; Wild et al. 1963), while Wild (1970) proposed to revert to the earlier terminology storm continuum. Section 16.4 of Kai et al. (1985) summarises the state-of-the art in the 1980s. Stationary type IV bursts demonstrate that non-thermal electrons are present in the corona for several hours in relationship with a flare and the liftoff of a CME. They are therefore a key element to investigate the restructuring of the solar corona in the aftermath of the CME. Kahler \& Hundhausen (1992) relate the electron acceleration to magnetic reconnection in the current sheets forming in the wake of a CME.

Since the above-cited early systematic studies of stationary type IV bursts the observational diagnostics have been considerably enriched: a large archive of imaging observations at radio wavelengths is available at frequencies up to above $400 \mathrm{MHz}$ with the Nançay Radioheliograph (NRH; Kerdraon \& Delouis 1997), and a number of spacecraft provide magnetograms of the photosphere and images of the corona at EUV wavelengths. But only case studies of individual stationary type IV bursts were published: Koval et al. (2016) analysed source positions over an extended frequency range down to the ionospheric cutoff, and Liu et al. (2018) argued that the electron cyclotron maser instability produces the emission, rather than the traditionally envisaged plasma emission processes. The new diagnostics are employed in the present work in the attempt of a systematic study using seven well-observed stationary type IV bursts. The aim is to identify the magnetic structures where stationary type IV bursts occur and their relationship with the parent flare and CME. The article starts with a detailed case study of the stationary type IV burst of the "Bastille day" event July 14, 2000. A description of the radio sources and their polarisation is given in Sect. 2, and the relationship with EUV signatures and the photospheric magnetic field is analysed. In Sect. 3, we summarise the results from a similar study of six other events. The details of these are given in the appendix. In Sect. 4, the results are discussed with emphasis on the magnetic configuration of the source, the mode of radio emission, the incomplete polarisation, and the energy of the radio-emitting electrons.

\section{An illustration: the July 14, 2000 eruptive event and its ICME}

\subsection{Overview of the "Bastille day" event}

We use the "Bastille day" flare and CME to illustrate our procedure. Structures of the EUV emission during this event were studied in Aschwanden \& Alexander (2001) and Chertok \& Grechnev (2005), the magnetic configuration by Yan et al. (2001), Zhang (2002), Török et al. (2018), and the CME by Andrews (2001). The decimetre-to-metre-wave radio emission was described in Caroubalos et al. (2001a), Maia et al. (2001), Klein et al. (2001) and Alissandrakis et al. (2019). The first image of the coronal mass ejection (CME) was seen by the Large Angle and Spectrometric Coronograph experiment (LASCO; Brueckner et al. 1995) of the Solar and Heliospheric Observatory (SoHO) at 10:54 UT with speed of $\sim 1700 \mathrm{~km} \mathrm{~s}^{-1}$ (CME LASCO catalogue ${ }^{1}$ ). The CME was a halo associated

\footnotetext{
1 https://cdaw.gsfc.nasa.gov/CME_list/
}

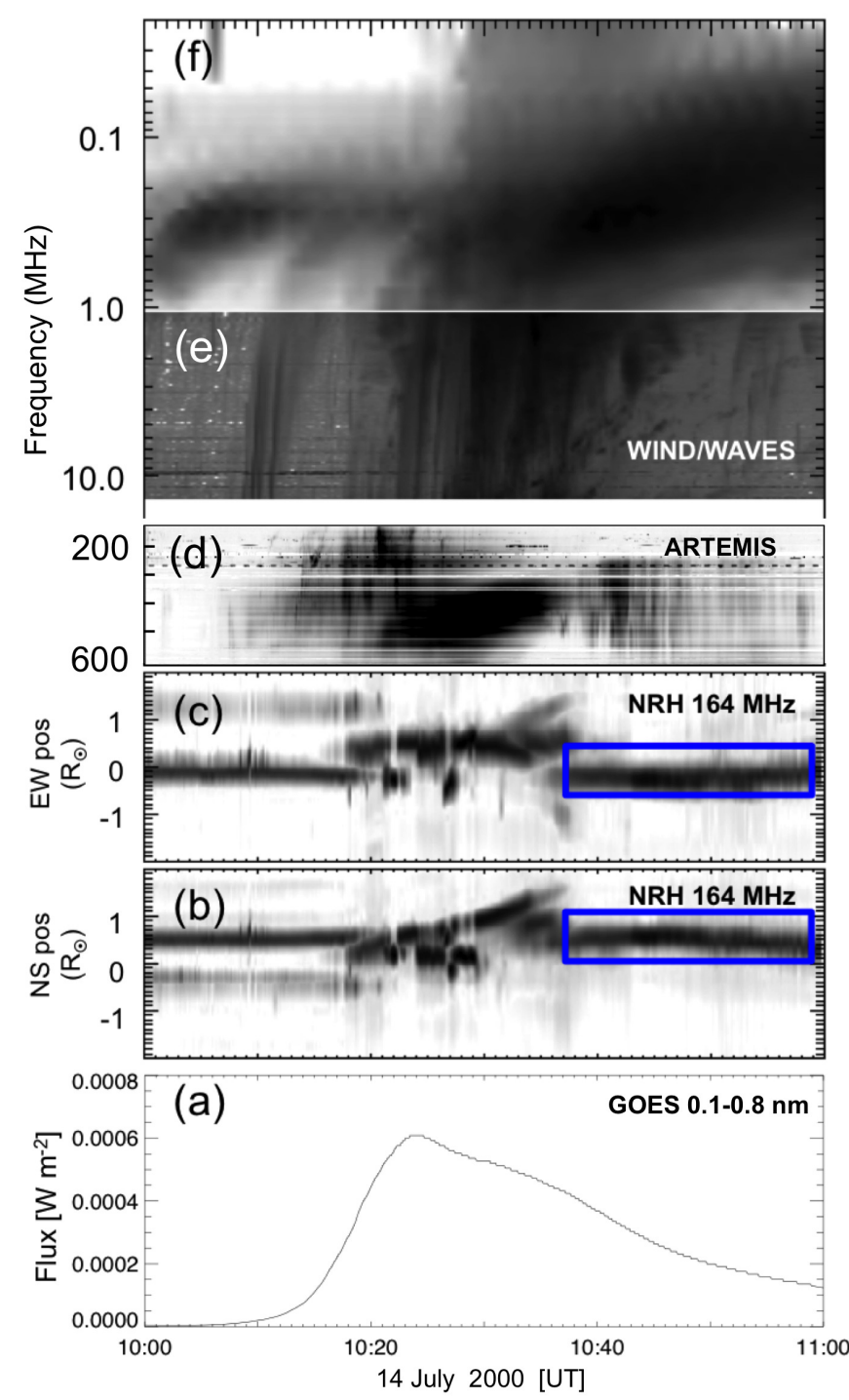

Fig. 1. Multifrequency plot of soft X-ray and radio emissions for the "Bastille Day Event". Panels show: (a) soft X-ray profile; $(b)$ 1D images at $164 \mathrm{MHz}$ projected on the solar north-south and $(c)$ east-west directions ( $y$ axis graded in solar radii, with respect to the centre of the solar disk); the stationary type IV burst (STIVB) is enclosed by the blue rectangles. $(d)-(f)$ : dynamic spectra between $650 \mathrm{MHz}$ and $10 \mathrm{kHz}$.

with a X5.7 class flare close to the central meridian $\left(\mathrm{N} 17^{\circ} \mathrm{W} 11^{\circ}\right)$ and with a filament eruption (Wang et al. 2006). A summary is listed in Table 1.

The time evolution of the soft X-ray and radio emission during the solar event is depicted in Fig. 1. The bottom panel a shows the soft X-ray flux in the $0.1-0.8 \mathrm{~nm}$ channel of the Geostationary Operational Environmental Satellite (GOES) satellite $^{2}$. Panels $d$ to $f$ display the dynamic spectra in the decimetre-to-kilometre wave range as observed by the groundbased ARTEMIS spectrograph (Caroubalos et al. 2001b) and the WAVES spectrograph (Bougeret et al. 1995) on the Wind spacecraft. Type III bursts occurred in the range $110-650 \mathrm{MHz}$ (Fig. 1d) during the early rise phase of the soft X-ray burst around 10:10 UT and continued in the interplanetary space as seen in the WAVES spectrum. A broadband emission that gradually drifted towards lower frequencies is recognised in the

\footnotetext{
2 http://WwW.nasa.gov/content/goes-overview/
} 
Table 1. Stationary type IV bursts (STIVB) and associated phenomena.

\begin{tabular}{|c|c|c|c|c|c|c|c|c|}
\hline Date & $\begin{array}{r}\mathrm{CME} \\
\text { 1st image } \\
{[\mathrm{UT}]} \\
(2)\end{array}$ & $\begin{array}{r}\text { Flare } \\
\text { onset } \\
\text { [UT] } \\
(3)\end{array}$ & $\begin{array}{r}\text { Flare } \\
\text { peak } \\
{[\mathrm{UT}]} \\
(4)\end{array}$ & $\begin{array}{r}\mathrm{AR} \\
\text { location } \\
(5)\end{array}$ & $\begin{array}{r}\text { EUV loop } \\
\text { arcade } \\
{[\mathrm{UT}]} \\
(6)\end{array}$ & $\begin{array}{r}\text { STIVB } \\
\text { onset } \\
\text { [UT] } \\
(7)\end{array}$ & $\begin{array}{r}\text { Average } \\
\text { polarisation } \\
(8)\end{array}$ & $\begin{array}{r}\text { Average } \\
\log \left(T_{\mathrm{B}}\right) \\
(9)\end{array}$ \\
\hline $2000 \mathrm{Jul} 14$ & $10: 54$ & $\begin{array}{r}10: 03 \\
(\mathrm{X} 5.7)\end{array}$ & $10: 24$ & N17 W11 & $10: 28 / 10: 38$ & $\begin{array}{l}\text { HF: } 10: 26 \\
\text { LF: } 10: 38\end{array}$ & $\begin{array}{l}\text { 164 MHz: } 86 \% \pm 8 \% \\
\text { 237 MHz: } 76 \% \pm 7 \% \\
\text { 327 MHz: } 60 \% \pm 7 \% \\
\text { 432 MHz: } 90 \% \pm 3 \%\end{array}$ & $\begin{array}{l}8.19 \pm 0.13 \\
7.77 \pm 0.21 \\
7.78 \pm 0.41 \\
7.98 \pm 0.41\end{array}$ \\
\hline 2001 Oct 09 & $11: 30$ & $\begin{array}{r}10: 46 \\
(\mathrm{M} 1.4)\end{array}$ & $11: 13$ & S30 E05 & 11:00/11:12 & $\begin{array}{l}\text { HF: } 10: 53 \\
\text { LF: } 10: 57\end{array}$ & $\begin{array}{l}\text { 164 MHz: } 67 \% \pm 4 \% \\
\text { 237 MHz: } 82 \% \pm 4 \% \\
\text { 327 MHz: } 46 \% \pm 3 \% \\
432 \mathrm{MHz}: 77 \% \pm 3 \%\end{array}$ & $\begin{array}{l}8.41 \pm 0.25 \\
8.17 \pm 0.18 \\
7.88 \pm 0.24 \\
7.65 \pm 0.22\end{array}$ \\
\hline 2007 May 19 & $13: 24$ & $\begin{array}{r}12: 50 \\
(\mathrm{~B} 9.5)\end{array}$ & 13:02 & N00 W05 & $12: 49 / 12: 52$ & $\begin{array}{l}\text { HF: } 12: 49 \\
\text { LF: } 13: 03\end{array}$ & $\begin{array}{r}\text { 151 MHz: } 102 \% \pm 6 \% \\
237 \mathrm{MHz}: 99 \% \pm 2 \% \\
327 \mathrm{MHz}: 92 \% \pm 3 \% \\
432 \mathrm{MHz}: 65 \% \pm 9 \%\end{array}$ & $\begin{array}{l}8.02 \pm 0.06 \\
7.21 \pm 0.06 \\
6.45 \pm 0.06 \\
5.79 \pm 0.09\end{array}$ \\
\hline 2008 Apr 26 & $14: 30$ & $\begin{array}{r}13: 50 \\
(\mathrm{~B} 3.8)\end{array}$ & $14: 08$ & N10 E10 & $13: 48 / 14: 00$ & $\begin{array}{l}\text { HF: } 13: 55 \\
\text { LF: } 14: 02\end{array}$ & $\begin{array}{r}\text { 151 MHz: } 84 \% \pm 19 \% \\
228 \mathrm{MHz}: 57 \% \pm 17 \% \\
327 \mathrm{MHz}: 24 \% \pm 16 \% \\
432 \mathrm{MHz}:-6 \% \pm 7 \%\end{array}$ & $\begin{array}{l}5.98 \pm 0.22 \\
5.64 \pm 0.19 \\
5.53 \pm 0.11 \\
5.59 \pm 0.09\end{array}$ \\
\hline 2010 Apr 03 & $10: 34$ & $\begin{array}{r}08: 50 \\
(\mathrm{~B} 7.4)\end{array}$ & $10: 00$ & S22 W29 & $\begin{array}{r}09: 48 / 10: 00 \\
(\text { EIT) } \\
09: 54 / 10: 14 \\
\text { (SWAP) }\end{array}$ & $\begin{array}{r}\text { HF: 09:50 } \\
\text { LF: }<10: 05\end{array}$ & $\begin{array}{r}\text { 151 MHz: } 83 \% \pm 12 \% \\
237 \mathrm{MHz}: 92 \% \pm 7 \% \\
327 \mathrm{MHz}: 87 \% \pm 19 \% \\
432 \mathrm{MHz}: 88 \% \pm 20 \%\end{array}$ & $\begin{array}{l}7.03 \pm 0.11 \\
6.67 \pm 0.10 \\
6.43 \pm 0.13 \\
6.59 \pm 0.09\end{array}$ \\
\hline 2012 Mar 04 & 11:00 & $\begin{array}{r}10: 29 \\
(\mathrm{M} 2.0)\end{array}$ & $10: 52$ & N18 E54 & $10: 32 / 10: 48$ & $\begin{array}{l}\text { HF: } 10: 38 \\
\text { LF: } 10: 50\end{array}$ & $\begin{array}{l}\text { 151 MHz: } 39 \% \pm 24 \% \\
228 \mathrm{MHz}: 51 \% \pm 20 \% \\
327 \mathrm{MHz}: 50 \% \pm 14 \% \\
432 \mathrm{MHz}: 53 \% \pm 15 \%\end{array}$ & $\begin{array}{l}7.80 \pm 0.35 \\
8.51 \pm 0.33 \\
9.15 \pm 0.46 \\
9.15 \pm 0.50\end{array}$ \\
\hline 2012 Jun 14 & $14: 12$ & $\begin{array}{r}13: 20 \\
(\mathrm{M} 1.9)\end{array}$ & $14: 30$ & S17 W00 & $13: 44 / 13: 52$ & $\begin{array}{l}\text { HF: } 14: 06 \\
\text { LF: } 14: 32\end{array}$ & $\begin{array}{c}\text { Eastern source }( \\
\text { 151 MHz: } 71 \% \pm 6 \% \\
228 \mathrm{MHz}: 88 \% \pm 7 \% \\
327 \mathrm{MHz}: 78 \% \pm 8 \% \\
432 \mathrm{MHz}: 64 \% \pm 25 \% \\
\text { Western source } \\
432 \mathrm{MHz}: 96 \% \pm 3 \%\end{array}$ & $\begin{array}{l}<0): \\
9.24 \pm 0.17 \\
9.06 \pm 0.07 \\
8.74 \pm 0.15 \\
8.49 \pm 0.27 \\
>0): \\
8.45 \pm 0.11\end{array}$ \\
\hline
\end{tabular}

Notes. Contents: date (Col. 1), time of the first observation of the CME by the LASCO coronagraph (Col. 2), flare onset time and the flare class (GOES; Col. 3), flare peak (Col. 4), active region location (Col. 5), first appearance of the loop arcade in EUV images (Col. 6), stationary type IV burst (STIVB) onset (Col. 7), average of polarisation degree (Col. 8) and the logarithm of the brightness temperature (Col. 9) of the STIVB at different frequencies.

ARTEMIS spectrum between 10:25 and 10:40 UT. During this time intense type III bursts were observed at lower frequencies $(1-10 \mathrm{MHz})$. Afterwards, a broadband emission continued with strong pulsations in the decay phase of the soft X-ray burst.

\subsection{Identification and localisation of radio sources after the flare}

Figures $1 \mathrm{~b}$ and $\mathrm{c}$ show one-dimensional radio images projected onto the solar north-south and east-west direction, respectively, taken by the Nançay Radioheliograph at $164 \mathrm{MHz}$, henceforth referred to as NRH (Kerdraon \& Delouis 1997). At a given time, the 1D image in a given direction is the scan computed by summing over all pixels along the orthogonal direction. The temporal succession of the resulting scans is then plotted as a grey-scale image. In order to show weak and strong sources, the scans are normalised individually. The 1D images plotted here therefore do not display the time evolution of the source brightness. Throughout the present work, we use NRH data with $10 \mathrm{~s}$ integration time, unless stated otherwise. This is sufficient to show structural changes of the stationary type IV radio sources. Spectral fine structure would need higher resolution, but is not considered here. Some plots use 2 min integration for clarity.

The 1D images show a noise storm prior to the eruptive event (before $\sim 10: 20 \mathrm{UT}$ ), followed by widespread emission with strong temporal and spatial variability between about 10:18 and 10:40 UT. A moving type IV burst is clearly visible as a source that moves north-westwards (towards solar north in Fig. $1 \mathrm{~b}$ and west in Fig. 1c). After $\sim 10: 35 \mathrm{UT}$ a single source located near the central meridian in the northern hemisphere became dominant until the end of the plot at 11:00 UT (enclosed within blue rectangles). It was actually still visible at the end of NRH observations near 15:20 $\mathrm{UT}^{3}$ at all frequencies observed by the NRH $(432,411,327,237$ and $164 \mathrm{MHz})$. This is the stationary type IV burst that we analyse in the present work.

The stationary type IV sources near the onset of the emission are overplotted in Fig. 2 at all frequencies on the image

3 See daily plots at http://secchirh.obspm.fr/ 


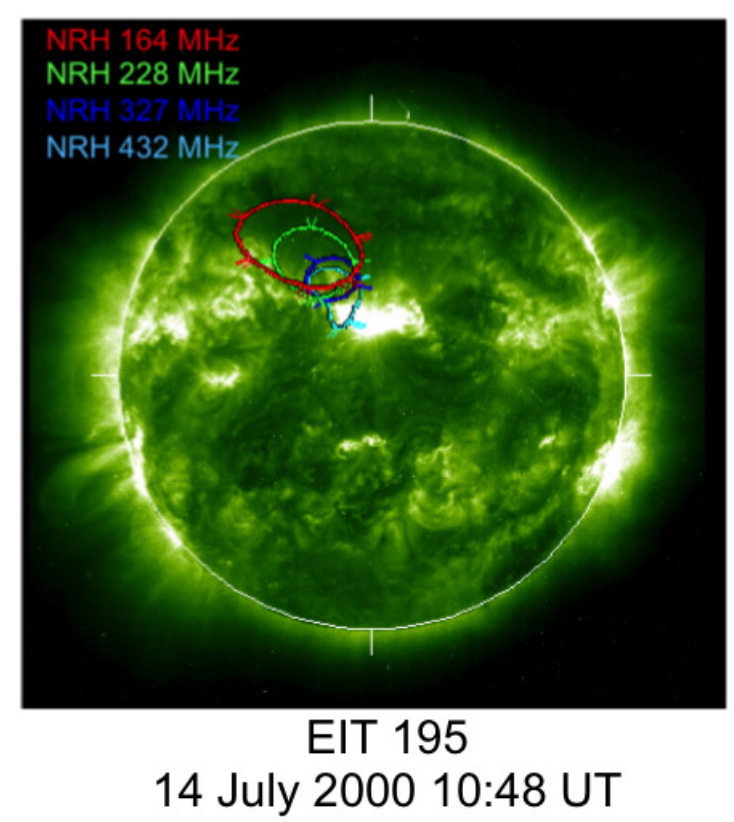

Fig. 2. Superposition of radio sources seen in Stokes parameter $I$ on an EIT image at $19.5 \mathrm{~nm}$ wavelength for the stationary type IV source on July 14,2000 . The contours of equal brightness temperature at $50 \%$ of the maximum are plotted in colours to represent different frequencies.

taken at $19.5 \mathrm{~nm}$ wavelengths by the Extreme Ultraviolet Imaging Telescope (EIT) aboard SoHO (Delaboudinière et al. 1995). The radio sources are represented by iso-intensity contours at $50 \%$ of maximum brightness. The radio sources are broad, and the contours reflect mostly the antenna beam. The NRH is a T-shaped array with a size of $3.2 \mathrm{~km}$ in the terrestrial eastwest direction, and $2.5 \mathrm{~km}$ in the north-south direction. However, the two-dimensional snapshot mapping uses only the inner $1.6 \times 1.25 \mathrm{~km}^{2}$ baselines, yielding a spatial resolution of typically $6^{\prime}$ at $150 \mathrm{MHz}$ (Kerdraon \& Delouis 1997).

The radio sources overlay the eastern part of the arcade of flare loops seen in EUV. In projection on the solar disk the radio sources were the farther away from the flare site, the lower the frequency. They formed a columnar structure with north-eastward orientation. The EUV arcade was seen at $17.1 \mathrm{~nm}$ (Aschwanden \& Alexander 2001) to brighten first in its western part, when metre wave radio emission was dominated by moving sources. The first flare ribbons in the eastern part were established between 10:11:09 UT and 10:28:06 UT, the first loops between 10:28:06 UT and 10:37:40 UT (their Fig. 7). The stationary type IV burst in the overlying corona was first seen at $432 \mathrm{MHz}(10: 26 \mathrm{UT})$ and appeared successively later at the lower frequencies (10:38 UT at $164 \mathrm{MHz})$. The appearance of the stationary type IV burst was hence closely connected in time and space to the appearance of the eastern flare loops in EUV.

Despite the widely used name stationary type IV burst, the source is not static, as shown in the movie accompanying this article. The dominant radio sources observed by the NRH are displayed by contours of equal brightness at $50 \%$ and $90 \%$ of the brightest point in each map. NRH data with two-minute integration time were used, and overlaid on the SoHO/EIT image at $19.5 \mathrm{~nm}$ wavelength taken near or before the time of the radio map. The contours are plotted in blue (411 and $432 \mathrm{MHz}$ ), green (327 MHz), yellow (237 MHz), and red (164 MHz). The first three images of the movie show the situation before the flare.
Radio emission came from two noise storms above the future flaring active region. The NRH images between 10:07:42 UT and 10:25:42 UT show multiple radio sources during the impulsive and early post-impulsive flare phase. The first image of the stationary type IV burst at frequencies above $400 \mathrm{MHz}$ is at 10:25:42 UT. The source is multiple in the next image. The columnar structure was visible since 10:29:42 UT, and was fully developed at 10:41:42 UT. A distinct second source was visible at high frequencies above the western end of the active region between 11:01:42 UT and 11:09:42 UT. A new source appeared in the southern hemisphere briefly at 11:49:42 UT. We consider in the following that new episodes of energy release occurred after 11:49 UT and stop our study of the stationary type IV emission at that time. A more detailed analysis of the later radio emission was given in Maia et al. (2001).

Between its appearance and 11:49 UT the columnar source underwent a series of systematic apparent motions: a northeastward bending between 10:33:42 UT and 10:45:42 UT, when especially the low-frequency sources were shifted away from the active region; between 10:49:42 UT and 10:59:42 UT the entire columnar structure swung back to the active region. This motion was accompanied by the appearance of a new source above $400 \mathrm{MHz}$ south of the previous one. Thereafter motions had smaller amplitudes, looking like a whirling motion of the structure outlined by the low frequencies around its low-coronal part marked by the high frequencies. This was perhaps not a real motion, but a change of internal structure, since the contours change shape, indicating the appearance of new sources closer to the active region. This is readily visible at frequencies above $400 \mathrm{MHz}$. A double source was observed after 11:44 UT. Its south-western part appeared like the high-frequency counterpart of the stationary type IV burst, located at that time near the centre of the arcade of flare loops in EUV. The north-eastern part may mark the residual of the previous emission or a reappearance of the noise storm. This source became the dominant and persistent source of the high-frequency emission after 11:58 UT, which we do not include in this analysis.

The presence of different structures could be consistent with the fine structure observed in the dynamic spectrum by ARTEMIS between 10:43 and 10:50 UT. A high cadence spectrum by the ARTEMIS-IV radio spectrograph in Caroubalos et al. (2001a) shows that this emission was a continuum with superposed broadband pulsations. Figure 3a displays the time evolution of the position of maximum intensity in NRH images at different frequencies between 10:58 UT and 11:55 UT to show the gradual displacement towards the centre of the underlying arcade of EUV loops. A temporary source is observed between 11:02 and 11:09 UT above the western part of the active region. Henceforth this source is labelled Sb. It was visible with an intensity below $50 \%$ of the peak at lower frequencies, as is shown in Fig. 4. It coexisted with the previous source. This double source persisted during some minutes (between $\sim 10$ and $25 \mathrm{~min}$ ), then $\mathrm{Sb}$ disappeared.

Between $\sim 11: 32$ UT and $\sim 11: 55 \mathrm{UT}$, the radio sources were located above the central region of the underlying arcade. The emission at $432 \mathrm{MHz}$ enclosed by the white circle in Fig. 3a is a persisting (11:44-12:48 UT) remnant at high frequencies of the initial columnar structure.

\subsection{Polarisation of the stationary type IV sources}

A relevant property of stationary type IV bursts is their circular polarisation. We analyse the sense and degree of the 


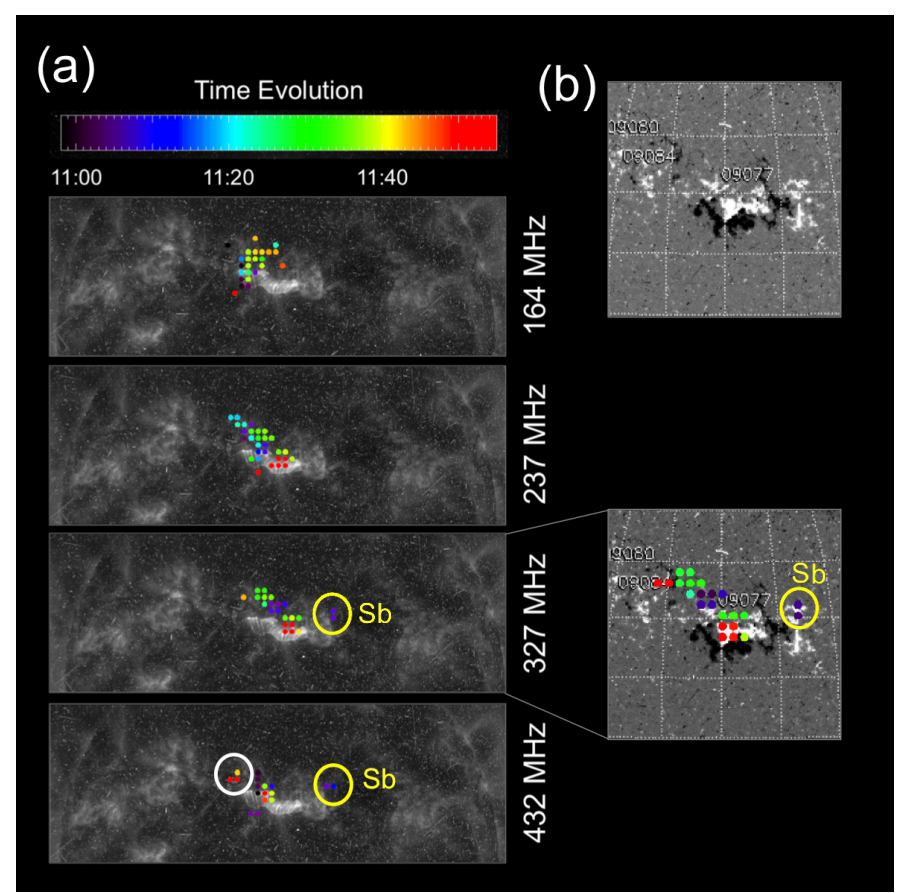

Fig. 3. (a) Time evolution (time is colour-coded) of the peak position on July 14, 2000 of radio sources in NRH images between 10:58 and 11:55 UT at four frequencies overploted onto EIT images and $(b)$ on the MDI magnetogram of the active region NOAA 9077.

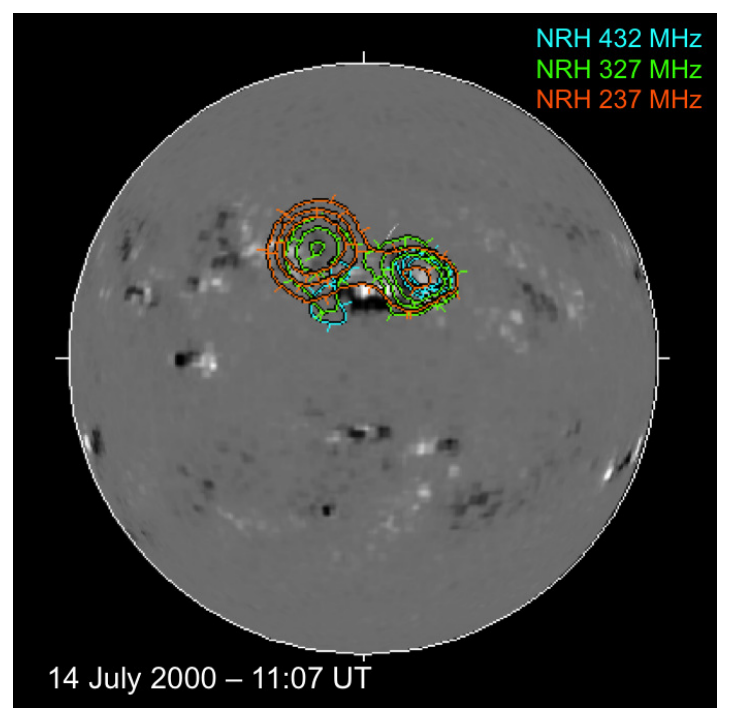

Fig. 4. Superposition of radio sources seen in Stokes parameter $I$ on an MDI magnetogram. Contours of equal brightness temperature at $50 \%$ of the maximum are plotted in different colours for different frequencies.

polarisation of the sources using maps of the Stokes parameters $I$ and $V$ observed by NRH at four different frequencies $(432,327$, 226 and $164 \mathrm{MHz}$ ).

The polarisation of the long-lived eastern columnar source of the stationary type IV burst, as shown in Fig. 4, was left-handed $(V>0$; see Kraus 1966) at all frequencies until 11:50 UT. The radio source is overlaid on the photospheric magnetogram taken by the Michelson Doppler Imager (MDI) aboard SoHO (Scherrer et al. 1995). The magnetogram of the flaring active region presents an extended region of positive magnetic polarity at the centre, surrounded by negative polarity at its eastern,
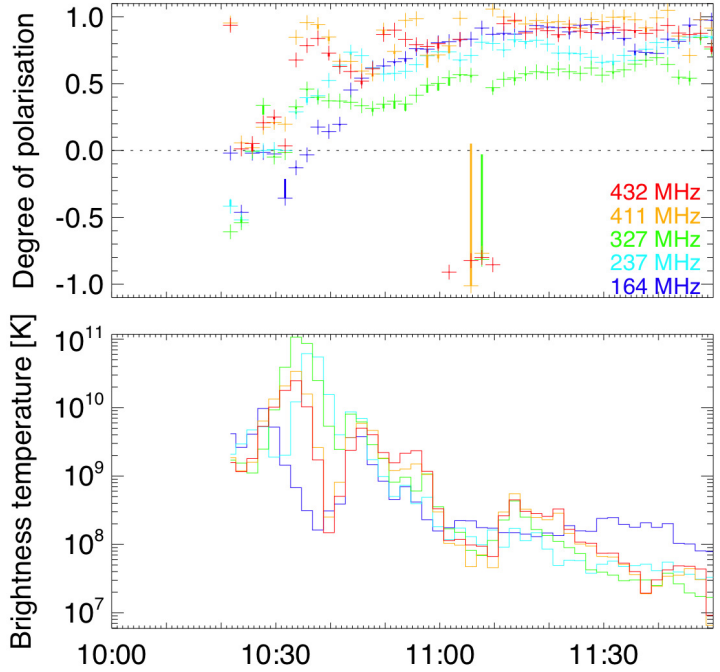

14 July 2000 [UT]

Fig. 5. Time histories of the polarisation degree (top) and brightness temperature (bottom) for the stationary type IV source on July 14, plotted with an integration time of $2 \mathrm{~min}$. The different colours represent the frequencies.

southern and western borders. As illustrated in Fig. 3b for $327 \mathrm{MHz}$, the high-frequency sources of the stationary type IV emission clustered in two groups, which were also identified in the movie. The main source (the south-western one), which appeared as the high-frequency counterpart of the columnar stationary type IV source, projects onto the region of northward (positive) magnetic polarity in the photospheric magnetogram. The north-eastern component had the same sense of circular polarisation until 11:50. It projects onto a region of weak fields with mixed polarities in the photospheric magnetogram, which does not allow us an unambiguous identification of the magnetic field orientation. However, judging from the location of the highfrequency sources of the columnar structure, the main source of the stationary type IV emission emitted in the ordinary mode. Because of the complex source structure this conclusion is tentative, and will have to be confronted with results for the other events.

The western source ( $\mathrm{Sb}$ in Fig. 3a) had the opposite, righthanded, polarisation. It is tempting to interpret the bipolar structure as evidence that the radio sources were located in the two legs of a large-scale magnetic structure oriented in the east-west direction, similar to Fig. 10 of Zhang (2002). But the comparison with the magnetogram contradicts this view. The western radio source projects onto a photospheric magnetic field directed towards the observer. The right-hand polarised emission of the western source hence must be in the extraordinary mode. Given the high frequency and the good correspondence of the source location with the northern magnetic polarity in the photosphere, it is not likely that this is a misidentification. Furthermore the two components did not display a common variation of their brightness temperature profiles. A correlation between them could have been taken as independent evidence on a common magnetic configuration. Because of its short lifetime, the western source is likely not a characteristic feature of the stationary type IV burst.

The time evolution of the dominant degree of circular polarisation and of the brightness temperature after the establishment of the entire columnar structure north-eastwards of the flaring active region (after $\sim 10: 46 \mathrm{UT}$ ) is shown in Fig. 5. The degree 
(a)

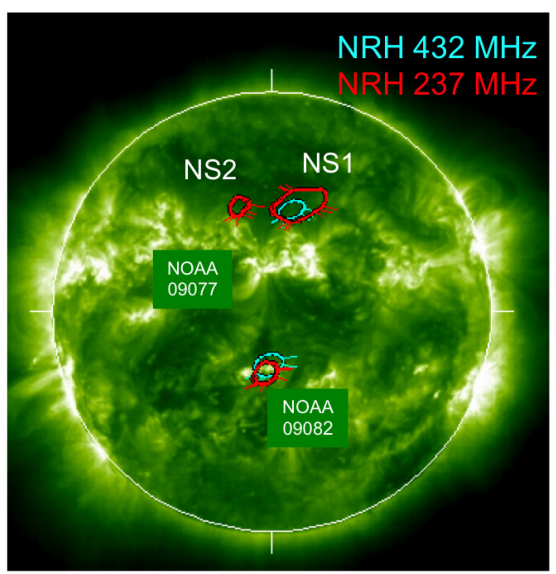

(b)

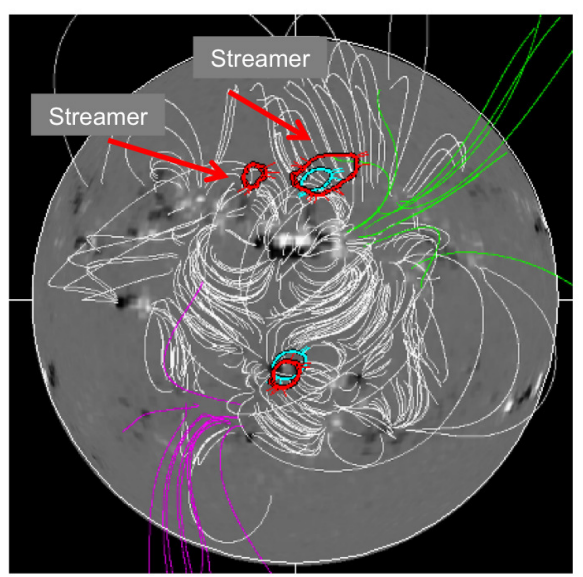

(c)

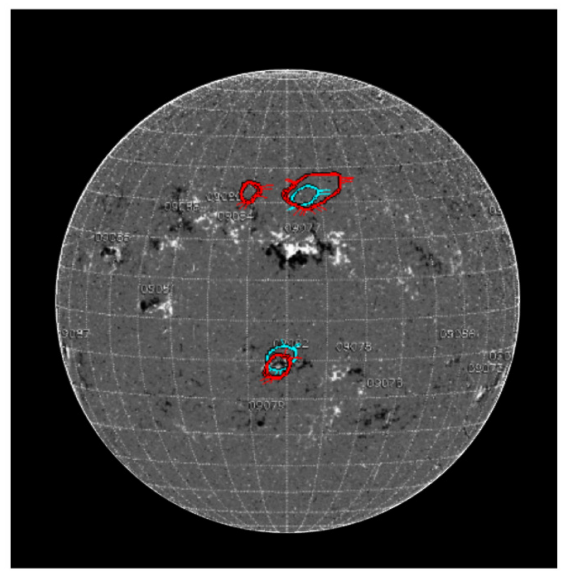

Fig. 6. $a$ : superposition of noise storm contours at $432 \mathrm{MHz}$ and $237 \mathrm{MHz}$ onto an EIT image at 09:50 UT on July 14, 2000. $b$ : superposition of the sources on the photospheric magnetogram (MDI) and coronal field lines inferred using the PFSS model by Schrijver \& De Rosa (2003). The magnetic configuration associated with the streamer regions are indicated by red arrows. The streamer associated with NS1 was examined by Török et al. (2018). $c$ : superposition of the radio sources on an MDI magnetogram.

of circular polarisation is calculated at the brightest point in the map of Stokes parameter $I$. This is done in an automated procedure. The source in total intensity $(I)$ in the one-dimensional scan through the point in the east-west direction is represented by a gaussian on top of a straight line. The straight line approximates the quiet Sun background, which was not always negligible compared with the non-thermal source. The gaussian is considered as the brightness temperature profile of the nonthermal source. The brightness temperature profile in $V$ is corrected for background distortions, which may occur because of calibration errors, by subtracting a third-order polynomial. The polynomial is determined by a least-squares fit, avoiding points that represent significant sources. The degree of polarisation is the ratio $p=I / V$ obtained from the corrected profiles. The stationary type IV source showed a gradual increase during the first tens of minutes to a stable value.

The average values of the degree of circular polarisation and their standard deviations, computed in the interval 11:10-11:50 UT, when the degree of polarisation had reached a stable value, are listed in Table 1 (Col. 8), together with the mean values of the logarithms of the brightness temperature (Col. 9). Since the radio sources were not resolved by the NRH, the brightness temperatures are averages over the antenna beam. An inspection of the images shows that the circular polarisation was not simple. The brightest points in the $I$ and $V$-maps were initially distinct and approached each other as the degree of polarisation rose. This suggests partially resolved or unresolved source components that coexisted with different degrees of circular polarisation. Figure 5 shows that the rise of polarisation at $327 \mathrm{MHz}$ and lower frequencies after 10:40 coincided with the decay of the brightness temperature from values of about $8 \times 10^{9} \mathrm{~K}$ to a few times $10^{8} \mathrm{~K}$, while the later phase of stable polarisation accompanied a slower decrease of the brightness temperature at levels around $10^{8} \mathrm{~K}$. At 411 and $432 \mathrm{MHz}$ the initial evolution was more complex, but resembled the lower frequencies after 10:45.

\subsection{The stationary type IV burst and the pre-existing noise storm}

Three noise storm sources were observed before the flare. Two were associated with the active region of interest here (labelled
NS1 and NS2 in Fig. 6a), another one with active region NOAA 09082 in the southern hemisphere. The two former noise storms were observed near the stationary type IV source, but with the opposite sense of circular polarisation. Figures $6 \mathrm{~b}$ and $\mathrm{c}$ show the photospheric magnetogram from SoHO/MDI, with coronal field lines superposed on Fig. 6b. The field lines were calculated with the Potential Field Source Surface (PFSS) model of Schrijver \& De Rosa (2003), available in the SolarSoft software package. Iso-intensity contours (50\% of the peak value) of the noise storm sources at 09:50 UT at $432 \mathrm{MHz}$ (light blue) and $237 \mathrm{MHz}$ (red) are overplotted onto both figures. A comparison with Fig. 3 shows that at these frequencies NS1 is well northwards of the stationary type IV source, while NS2 is located close to the initial position of the stationary type IV source. In projection on the magnetogram (Fig. 6c) the noise storm sources lie above regions of negative (southward) magnetic polarity. The right-handed circular polarisation is hence consistent with radio emission in the ordinary mode. The degree of polarisation is about $90 \%$ (164 and $237 \mathrm{MHz}$ ), 80\% (327 MHz), 95\% (411 and $432 \mathrm{MHz})$.

\section{Results on six further stationary type IV continua}

The event on July 14, 2000 presents a number of properties reported for stationary type IV bursts in the literature: the dominant source structure is columnar with a single sense of polarisation, which by comparison with the photospheric magnetogram we identify as the ordinary mode. The high degree of polarisation is consistent with the general idea of fundamental plasma emission. The radio emission starts with an increasing delay at decreasing frequency, out of a complex source configuration including moving type IV sources. In addition, it could be shown that the establishment of the stationary type IV emission was related in time to the development of an arcade of flare loops seen in EUV images. A number of features observed in addition may perturb this simple picture. At certain times additional sources occur, forming a complex structure especially, but not exclusively, at higher frequencies. The possible evidence of highly polarised emission in the extraordinary mode located at the opposite extreme of the active region adds complication to the usual picture of fundamental plasma emission. 
In order to go beyond particularities of an individual event, especially an exceptional eruptive one like July 14, 2000, we selected six additional stationary type IV continua for a systematic study. To compare the radio source positions with the photospheric magnetic field, and also to minimise possible distortions of radio wave polarisation by propagation effects, we focussed on events that occurred on the solar disk and were observed across the entire $150-450 \mathrm{MHz}$ band covered by the NRH. For comparison we included one limb event on March 4, 2012. We conducted a detailed analysis of EUV images, photospheric magnetograms and radio data to identify and locate the radio sources. Each event is described in the appendix.

Characteristic times of the radio, soft X-ray and EUV emissions of the events are listed in Table 1. Columns 3 and 4 give the start and peak times of the soft X-ray burst in the $0.1-0.8 \mathrm{~nm}$ channel of GOES, respectively. The flare location is listed in Col. 5. Column 6 gives the time of the first appearance of an arcade of flare loops in coronal EUV lines, preceded by the time of the last image where the arcade was not seen. This information is taken from overview movies of EUV images from SoHO/EIT, the Extreme Ultaviolet Imager (EUVI; Wuelser et al. 2004) aboard the Solar-Terrestrial Relations Observatory (STEREO) mission, the Sun watcher using APS detectors and image processing (SWAP) imager aboard the Project for Onboard Autonomy (Proba2) satellite (Seaton et al. 2013) or the Atmospheric Imaging Assembly (AIA) aboard the Solar Dynamics Observatory (SDO; Lemen et al. 2012), depending on the event. The seventh column lists the start time of the stationary type IV emission. It can often not be reliably identified, because early manifestations may be hidden in the complex of previous radio sources. The quoted onset is the instant when we are sure to see for the first time the later dominating stationary source. Finally, the average values of the polarisation degree and the logarithm of the brightness temperature in the phase where the polarisation is stable are listed in Cols. 8 and 9, respectively.

The results of this analysis are summarised as follows:

1. The stationary type IV continua analysed in this work were observed during eruptive flares, associated with CMEs in coronagraphic images and with arcades of flare loops in EUV. CMEs in our sample were typically fast $\left(500-1700 \mathrm{~km} \mathrm{~s}^{-1}\right)$ and wide ( $100^{\circ}$ to halo). The associated flares ranged from weak (B3.8) to strong (X5.7). The association with CMEs is well known in the literature.

2. Timing of stationary type IV emission:

(a) In all studied cases stationary type IV continua were preceded by complex radio sources, which comprised moving type IV bursts. The stationary type IV burst appeared afterwards, but in general before the soft X-ray peak.

(b) The lower the observing frequency, the later the start time of the stationary type IV emission. A typical time lapse between the first appearance at frequencies above $400 \mathrm{MHz}$ and at $150 \mathrm{MHz}$ is $10 \mathrm{~min}$ (see Col. 7 in Table 1).

(c) The stationary type IV bursts started near the appearance of arcades of flare loops in coronal EUV emission (Col. 6 of Table 1). While the cadence of EUV instruments and the uncertainty of the actual start of the radio emission did not allow us to establish a clear timing association, the two phenomena were found to start together within ten to twenty minutes.

3. Structure and location of stationary type IV sources:

(a) Stationary type IV bursts have one dominant source with a columnar structure. In some events there is evidence at times for unresolved or partially resolved spatial structure, or for a second columnar structure appearing during the event at all or part of the observed frequencies (July 14, 2000; June 14, 2012; April 26, 2008; April 3, 2010).

(b) In events where the EUV arcades were extended (July 14, 2000; October 9, 2001; April 3, 2010; June 14, 2012) the stationary type IV sources were observed above one or, in the case of the double columnar structure on June 14, 2012, both extremities of the arcades.

(c) In two cases the type IV sources lay above dimmings in EUV images (April 3, 2010 and June 14, 2012). But this is not always the case (April 26, 2008).

(d) The sources of stationary type IV emission are not static, but show apparent movements. These movements may be real, revealing the expansion of large-scale magnetic field structures that host the radio sources. But unresolved or partially resolved fine structure of the radio sources could also point to a release of radio-emitting electrons onto different coronal magnetic field lines in the course of an event.

(e) In the only case with a bipolar structure (June 14, 2012) the brightness temperature profiles show a correlation with a delay of $36 \mathrm{~s}$. If ascribed to non-thermal electrons travelling from one source to the other, this delay implies an energy of about $20 \mathrm{keV}$.

4. Circular polarisation of the radio emission:

(a) With the exception of the limb event (March 4, 2012) the stationary type IV radio emission is found to be strongly polarised. But even when the polarisation is strong, it is most often not total. The degree of circular polarisation may vary with frequency. On April 26, 2008 the high-frequency emission is unpolarised, whilst the low-frequency emission is strongly polarised. The limb event was partially polarised during a few tens of minutes, but the polarisation then decreased to low values during most of the observed event duration.

(b) The columnar sources of stationary type IV bursts are polarised in one sense. Weakly polarised or unpolarised substructure was frequently observed.

(c) A persistent bipolar structure was found only in one case (June 14, 2012).

(d) In cases where the high-frequency part of the columnar sources, which is expected to be at lower altitude than the low-frequency sources, projected onto a clearly defined magnetic polarity in the photosphere, the sense of circular polarisation implied radio emission in the ordinary mode. This is especially clear for the bipolar source of June 14, 2012, where the oppositely polarised branches are found in magnetic fields with opposite directions. The extraordinary emission identified in a temporary source on July 14, 2000 is a unique case in our sample.

(e) In general the polarisation increased within some tens of minutes from zero or moderate values at the beginning to high values or even total polarisation. Only on April 3, 2010 was the emission fully polarised since the beginning. The increase of polarisation was accompanied by different evolutions of the brightness temperature: a correlated increase was only observed on October 9, 2001. The polarisation increased while the brightness temperature faded on July 14, 2000 and on May 19, 2007. The brightness temperature was observed to be stable or to decrease slowly during the phase of stable polarisation.

(f) The logarithm of the spatially averaged brightness temperatures in the phase of stable polarisation ranges from 6 to 9.2, generally decreasing with increasing frequency.

(g) Stationary type IV bursts that occurred in active regions with a pre-existing noise storm were found to have the opposite sense of circular polarisation as the noise storm on July 14, 2000 and October 9, 2001. This could be shown to be 
consistent with the magnetic field configuration: noise storms and stationary type IV bursts were located in different magnetic field configurations, and both emissions were consistent with radio emission in the ordinary mode.

\section{Discussion}

We studied a set of seven stationary type IV bursts in the frequency range $150-450 \mathrm{MHz}$. The core of the analysis used observations with the Nançay Radioheliograph in conjunction with EUV images and photospheric magnetograms from the SoHO, STEREO, SDO, and Proba2 missions. Many results confirm earlier knowledge, but some bring new insight, especially thanks to the extent to higher frequencies than in previous works.

\subsection{Source location and polarisation}

The basic spatial structure of a stationary type IV burst is columnar: sources at different frequencies are aligned, with their positions dispersed as a function of frequency, and are monopolar or unpolarised. The columnar structure of stationary type IV bursts is well known in the literature (Sect. 16.4 of Kai et al. 1985).

Bipolar sources at a given frequency are seen only in exceptional cases (June 14, 2012) or sporadically, during rather short time intervals as compared to the overall duration of the emission (July 14, 2000; April 26, 2008).

The usual single columnar structure is located at one extremity of the arcade of flare loops in EUV images. We conclude from the observations that the stationary type IV continuum is emitted in one leg of the flux rope forming the core of the CME. We exclude a location in the arcade of flare loops, because in that case one would expect the radio sources to be elongated along the arcade. This is not found in the events where an extended arcade is observed. If the radio source were in the arcade, one would also expect the two opposite polarisations from either side of the line of magnetic field reversal to cancel each other through the broad interferometric beam. The expansion of the flux rope and ensuing decrease of the ambient density can explain the delayed onset of the low-frequency emission, which is attributed to a plasma emission process, and is directly related to the ambient electron density.

This finding is more specific than earlier reports, which located the radio source close to the flare positions (Wild 1969; Kai et al. 1985). It is consistent with the location in the legs of CMEs reported in case studies by Gergely et al. (1979), Lantos et al. (1981), Gary et al. (1985), and more recently by Koval et al. (2016). The two parts of the bipolar columnar source on June 14, 2012 were located on either side of the flaring active region, projecting onto temporary dimmings in EUV images. This confirms the location of the radio sources in the legs of the erupting flux rope, since transient EUV dimmings are regions from which plasma is removed during a CME (e.g. Hudson et al. 1996; Zhukov \& Auchère 2004; Webb \& Howard 2012).

In strong events like July 14, 2000, the radio source looks more complex, and it undergoes systematic shifts with respect to the arcade of flare loops in the course of the event. The movements could be understood as the consequence of the complexity of the magnetic flux rope, as shown in the numerical model of Török et al. (2018). This model represented the erupting magnetic field by multiple flux rope structures. The radio source motion could be considered to reflect successive emission from different flux rope sections, instead of a physical motion of a single magnetic structure. In any case the observations of the entire sample of events give evidence on the location of the stationary type IV sources in the flux rope structure, rather than within the flare-loop arcade.

The correlated variation of brightness temperatures in the double columnar source on June 14, 2012 gives us a unique chance to estimate the overall extent of the magnetic structure hosting the radio emission. The estimated heliocentric distance of $3 R_{\odot}$ based on the extent of the CME core is again consistent with emission in the erupting flux rope. Stationary type IV bursts at comparable heliocentric distances were directly imaged by the Ukrainian UTR-2 telescope (20-30 MHz; Koval et al. 2016).

\subsection{Evidence on fundamental plasma emission}

Our observations confirm the well-known fact that stationary type IV sources, when seen on the disk, are in general strongly circularly polarised. Whenever the comparison between the sense of circular polarisation of the radio emission with the polarity of the underlying photospheric magnetic field allowed us a clear identification, the dominant radio emission was found in the ordinary mode. The extraordinary emission identified on July 14, 2000 is an exception, and its occurrence is limited in time. This result could be obtained because the studied stationary type IV bursts extended to high frequencies (above $400 \mathrm{MHz}$ ), which means to lower altitudes, than in most previous analyses. Projection effects therefore introduced less ambiguity than in earlier studies, and were further reduced by potential-field extrapolations into the corona.

While the ordinary-mode polarisation of stationary type IV bursts is textbook knowledge, it was so far established statistically, using the additional hypothesis that the magnetic polarity in the source is that of the leading or the dominant spot of the parent active region, and by analogy with noise storms, where the ordinary-mode polarisation had also been confirmed by the location above appropriately oriented magnetic fields in the photosphere (Dulk \& Nelson 1973; Habbal et al. 1989). Our observations give clear examples that the leading-spot hypothesis may be misleading (see also Stewart 1985). In several cases the stationary type IV source was located in the trailing part of the active region. Furthermore, in two cases the sense of circular polarisation of the stationary type IV burst was found opposite to that of a noise storm in the same active region, which is clearly inconsistent with expectations from the application of the leading-spot hypothesis to the two types of emission. The detailed identification of the magnetic structure hosting the radio source removes the ambiguity and shows that both the stationary type IV continuum and the noise storm were emitted in the ordinary mode, but that their sources were located in magnetic structures with opposite polarities.

The high polarisation of stationary type IV bursts in the ordinary mode is inconsistent with incoherent gyrosynchrotron emission. This mechanism had been suggested in a recent case study (Morosan et al. 2019), but the event under consideration occurred before a moving type IV burst, unlike the common stationary type IV continua. Emission from sources without substantial motion is in fact a common aspect of another kind of type IV burst, called a flare continuum (Wild 1970; Pick 1986). The high polarisation of the classical stationary type IV burst as studied in the present paper rather suggests a collective emission process, as expected for a population of trapped electrons in a dilute plasma, such as the leg of a magnetic flux rope in the corona. The most widely used models are based on spontaneous emission of upper hybrid waves and their conversion to radio waves by coalescence with low-frequency waves. These models were primarily developed for broadband continua in noise 
storms (Melrose 1980; Benz \& Wentzel 1981; Wentzel et al. 1986), and are also applicable to stationary type IV continua. They imply constraints on the brightness temperature that we further discuss below (Sect. 4.4).

If plasma emission arises from an instability of a trapped electron population, a strong harmonic component of the resulting radio emission is usually expected (Melrose 1980, 2017). This is inconsistent with observations of complete circular polarisation. Pure ordinary mode emission could, however, come from an electron cyclotron maser instability. In their comparison of electron cyclotron masers in different parameter ranges, Sharma \& Vlahos (1984) showed that electromagnetic waves in the ordinary mode will grow fastest if the ratio of plasma frequency to cyclotron frequency is between 0.35 and 1 . While common models of the corona assume weaker magnetic fields, these values of the characteristic frequencies are consistent with model-dependent estimates in and above some solar active regions (Régnier 2015; Morosan et al. 2016). It is not clear, however, if such parameters can be found in an extended height range as in the case of the stationary type IV emission between 450 and $150 \mathrm{MHz}$. The exact values of the parameters delimiting the ranges where different wave modes are predominantly amplified by the electron cyclotron maser instability are somewhat modeldependent (Sharma \& Vlahos 1984; Stupp 2000).

In conclusion, the observed sense of circular polarisation of stationary type IV continua is consistent with the indirect production of escaping electromagnetic waves in the ordinary mode via upper hybrid waves, as generally assumed for plasma emission models, and possibly with the direct production of electromagnetic waves in the ordinary mode by an electron cyclotron maser instability.

\subsection{How to understand partially polarised fundamental plasma emission?}

While stationary type IV bursts are often strongly circularly polarised, they seem to be rarely fully polarised (see also Robinson 1978, Fig. 4). This is often attributed to the peculiar location near the limb and the ensuing long travel path of the radio waves through the corona and the possible depolarisation, which may then occur. But our study shows that partially and weakly polarised stationary type IV emission may come from the solar disk. McCauley et al. (2019) reported a similar result for noise storm continua.

Full polarisation is expected for fundamental plasma emission if the radio waves are generated below the cutoff frequency of the extraordinary mode. The frequency of upper hybrid waves, $\omega_{\mathrm{uh}}=\sqrt{\omega_{\mathrm{pe}}^{2}+\omega_{\mathrm{ce}}^{2}+3 k^{2} V_{\mathrm{e}}^{2}}$, where $\omega_{\mathrm{pe}}$ is the electron plasma frequency, $\omega_{\mathrm{ce}}$ the electron cyclotron frequency, and $V_{\mathrm{e}}$ the thermal electron speed, is expected to be below the cutoff, except for rather high wave numbers $k$, which means low phase speeds (typically less than ten times the thermal electron speed, which is about $4800 \mathrm{~km} \mathrm{~s}^{-1}$ in a plasma at $1.5 \mathrm{MK}$ ). Since our travel time estimate on June 14, 2012 implies much higher speeds of about $0.3 c$, wave-particle resonances will occur only for phase speeds well above the thermal speed. The frequency increase by a coalescence process of the upper hybrid waves with ion sound waves or lower hybrid waves is too small to change this argument. One would therefore expect fully polarised emission in the ordinary mode, which is the classical situation for noise storm continua. Melrose (2017) concluded that the only way to understand partially polarised fundamental plasma emission is depolarisation of the originally fully polarised radio waves during their propagation through the corona.

A model for the temporary depolarisation of radio waves was described by Robinson (1985) in the attempt to explain the gradual increase of the polarisation in the early phase of a stationary type IV burst. He proposed the temporary creation of a turbulent region on the line of sight, where radio waves would travel through many small-scale quasi-transverse regions that produce mode coupling and a net depolarisation. This is plausible, since the radio emission has to travel through plasma lifted by the CME. The gradual disappearance of this region, as the CME expands, would explain the gradual increase of circular polarisation, and the persistence of a residual might explain why the polarisation of the stationary type IV emission does not become complete. But in order to understand why one of the components of the bipolar source on June 14, 2012 was fully polarised, while the other was partially polarised, one would have to invoke a spatially limited screen shielding one source during several tens of minutes, but not the other source. This seems artificial. A similar problem might occur with the explanation of a strongly frequency-dependent degree of polarisation as on April 26, 2008.

Robinson (1985) discarded the idea of a gradual transition between initial unpolarised harmonic plasma emission and strongly polarised fundamental plasma emission. One argument is that one should expect a systematic decrease of the source height as the emission changes from harmonic (greater height) to fundamental at the same frequency. Another argument is that the gradual increase of fundamental emission must imply the gradual increase of the level of low-frequency waves, with which the upper hybrid waves coalesce to generate a radio wave at the fundamental. Robinson argued that this would imply any increase of the degree of polarisation to go along with an increase of the overall brightness temperature. He did not see observational evidence for this, and in our sample only one event (October 9, 2001) shows this behaviour.

Robinson considered radio emission in a flux tube, where the density decreases with height, but is uniform in the perpendicular direction. In contrast our observations show unresolved fine structure of the radio sources, where weakly polarised (or unpolarised) and strongly polarised sources coexist within the antenna beam. This observation implies a fibrous coronal structure, which is indeed to be expected from EUV images. Any upwards or downwards motion within individual fibers might then be washed out by the antenna beam. One might also envisage that in such a flux tube some fibers favour fundamental emission, and others harmonic emission. Harmonic emission should be natural in the presence of a loss-cone distribution, since the amplified upper hybrid waves are expected to be more or less isotropic in the plane perpendicular to the magnetic field. Upper hybrid waves should then directly be able to coalesce nearly "head-on" into a radio wave (harmonic emission), while for a beam instability a separate process must reflect Langmuir waves to make them interact "head-on". Ion sound waves exist only when the electron temperature exceeds the ion temperature. That could justify the action of the process in individual fibers, where a gradual rise of the level of ion sound waves could explain the gradual increase of circular polarisation. It would be accompanied by the increase of the brightness temperature of the fundamental radio emission, but this rise might be masked by the independent evolution of the harmonic emission. While we have no independent evidence that this actually happens, the observation that the increase of circular polarisation in the early phase of a stationary type IV burst occurs together with the gradual 
disappearance of weakly polarised sources is a new finding that deserves consideration in any context of interpretation.

\subsection{Energy of the radio-emitting electrons and implications on the radio emission process}

The bipolar source on June 14, 2012 gave us a unique opportunity to estimate the energy of the radio-emitting electrons, through the interpretation of the observed time delay between the two sources as the travel time of a single population of nonthermal electrons. A minimum requirement for the electrons to emit radio waves in the two legs of a magnetic flux rope is that they survive Coulomb collisions with thermal electrons. For a quantitative estimate we consider the simple magnetic structure devised in Appendix F, made up of radial ascending and descending branches from the base of the corona to a heliocentric distance of $3 R_{\odot}$ and an arc over $14^{\circ}$ connecting them. The average energy loss of an electron with energy $E$ and speed $v$ by Coulomb collisions with thermal electrons of density $n_{\mathrm{e}}$ is given by Brown (1972):

$$
\frac{\mathrm{d} E}{\mathrm{~d} t}=-\frac{C}{E} n_{\mathrm{e}} v
$$

(cgs units), where $C=2 \pi e^{4} \Lambda, e$ is the elementary charge, $\Lambda \simeq 20$ the Coulomb logarithm. We use an isothermal hydrostatic density model,

$n_{\mathrm{e}}(r)=n_{\mathrm{e}}\left(R_{0}\right) \exp \left(-\frac{R_{0}}{H\left(R_{0}\right)}\left(1-\frac{R_{0}}{r}\right)\right)$,

with a reference heliocentric distance $R_{0}$. The scale height $H\left(R_{0}\right)=H_{\odot}\left(R_{0} / R_{\odot}\right)^{2}$, where $H_{\odot}$ is the scale height at the base of the corona. We assume a temperature of $1.5 \mathrm{MK}$. Since the stationary type IV burst is observed up to $445 \mathrm{MHz}$, and because we interpret the emission as fundamental plasma emission, the electron density at the foot of the flux rope must be at least $2.46 \times 10^{9} \mathrm{~cm}^{-3}$. With these parameters an electron with initial energy $30 \mathrm{keV}$ travels along the entire flux rope, reaching the opposite footpoint with an energy of about $26 \mathrm{keV}$. It can be mirrored back and forth several times. An electron with initial energy $10 \mathrm{keV}$ is thermalised on the ascending branch of the flux rope, and cannot contribute to the emission from the other source. The consideration of collisional lifetimes hence leads us to conclude that electrons must have energies above $10 \mathrm{keV}$ to travel from one branch of the magnetic flux rope to the other. This is consistent with the estimate from the correlation analysis of the brightness temperature time profiles in the event June 14, 2012. It is also consistent with energies derived from models of spontaneous plasma emission of radio continua (Wentzel 1986).

In the extended magnetic flux rope that we inferred to host the radio sources, only sufficiently energetic electrons would be able to bounce repeatedly between opposite footpoints. The limitation of the electron energy could hence explain why most stationary type IV sources have only one persistent branch. The extended duration of the stationary type IV emission cannot be understood by trapping, but requires the quasi-continuous acceleration of electrons throughout the radio event. This goes along with the continued formation of flare loops during several hours.

In the models of Spicer et al. (1982) and Vlahos et al. (1982), lower hybrid waves generated in the sheath between the outwards moving loop or flux rope and a shock wave ensure not only the generation of radio waves, but also the acceleration of the electrons producing the upper hybrid waves. Electron acceleration in the current sheets behind the flux rope was advocated on observational grounds by Kahler \& Hundhausen (1992). In both scenarios one may wonder why the electrons are only released into one leg of the flux rope. The post-CME current sheet extends along the entire arcade, but in the example of July 14, 2000 the flare loops formed together with the start of the stationary type IV continuum were indeed localised in the eastern part of the arcade. The asymmetric radio source would then reflect the spatial distribution of the reconnection process. Alternatively magnetic reconnection could occur between the leg of the expanding flux rope and the ambient corona. This process was investigated with numerical simulations by Masson et al. (2019) to explore the escape of particles from the flux rope. It might also give rise to particle acceleration. This scenario was actually inferred to operate by Kerdraon et al. (2010) and Salas-Matamoros et al. (2016) in two of the events studied in the present work (May 19, 2007 and April 26, 2008).

Spontaneous emission from a distribution of non-thermal electrons that are clearly separated from the thermal background through a gap ("gap distribution") would produce fundamental plasma emission with a negligible contribution to the harmonic (Wentzel 1985). The gap distribution could be rapidly generated by collisional losses of electrons around $1 \mathrm{keV}$ in the flux rope. The ambient electron densities derived from the radio observations are hence consistent with models of spontaneous plasma emission. The expected brightness temperature of the radio waves is comparable to the temperature of the energetic electrons, i.e. $4 \times 10^{8} \mathrm{~K}$ for electrons of $40 \mathrm{keV}$. Such energies are enough to allow electrons to bounce. Higher brightness temperatures should therefore be observed in bipolar sources. The event in our sample with the highest brightness temperature (June 14, 2012) has indeed a bipolar source.

As an alternative, a loss-cone instability of the trapped electrons will produce brighter fundamental emission, together with radio waves at the harmonic (Melrose 1980; Wentzel 1986). Such a distribution needs several bounce periods to be established, which, as discussed above, requires near-relativistic energies when the flux rope is extended. It may perhaps be a means to explain mixed sources with strong and weak polarisation, where the weakly polarised harmonic emission would dominate early in the event. This is consistent with high brightness temperatures early in the type IV continuum, when the emission is partially polarised.

\subsection{Conclusion}

The key findings of the present study of seven stationary type IV continua (also called storm continua) are the following:

Stationary type IV continua are emitted in one leg - in one case by a persistent bipolar source in both legs - of the erupting magnetic flux rope of the CME. From the correlation of brightness temperature profiles in the two components of the exceptional bipolar event we inferred that the radiating electrons had energies of a few tens of keV. We showed that such energies are sufficient to enable electrons to bounce back and forth along the extended flux rope, but that Coulomb collisions would prevent electrons with lower energies (below about $15 \mathrm{keV}$ in our estimate) from doing so. The upper limit of the energy could explain why most stationary type IV sources are restricted to one leg of the flux rope. A continuous acceleration of these electrons is in any case necessary to maintain the radio emission over more than a few tens of seconds.

The comparison of radio maps in Stokes parameters $I$ and $V$ with photospheric magnetograms and their extrapolation into the corona allowed us to confirm fundamental plasma emission 
in the ordinary mode as the radiation mechanism of stationary type IV sources. This result is consistent with previous work, but does not rely on the hitherto used hypothesis that the magnetic field polarity in the radio source is determined by the leading or dominant sunspot in the active region. This hypothesis would be misleading or inconclusive in our data set, where noise storms and stationary type IV continua from the same active region were observed to have opposite senses of polarisation.

The circular polarisation was in general found to be strong, but not total. The observations presented in this work show that the radio sources are not uniform, but comprise partially resolved and probably unresolved substructure with different polarisation. We propose to explain partial polarisation by the coexistence of fundamental and harmonic plasma emission in different substructures within the antenna beam.

Acknowledgements. This work could not have been conducted without the generous data provision of numerous instruments: the EIT, MDI, and LASCO instruments aboard SoHO (SOHO is a project of international cooperation between ESA and NASA); the SECCHI instrument suite aboard STEREO (NASA); the AIA and HMI instruments aboard SDO (courtesy of NASA/SDO and the AIA EVE, and HMI science teams); the SWAP instrument aboard Proba2 (ESA SWAP is a project of the Centre Spatial de Liège and the Royal Observatory of Belgium funded by the Belgian Federal Science Policy Office, BELSPO); the WAVES spectrograph on the Wind mission (NASA, PI LESIA, Paris Obs.); Kanzelhöhe Observatory (University of Graz); radio spectrographs ARTEMIS (operated by the University of Athens), NDA and ORFEES (Nançay radio astronomy station of Paris Observatory with support by the French research agency CNRS/INSU) and the Nançay Radioheliograph. We made frequent use of the SoHO/LASCO CME catalogue generated and maintained at the CDAW Data Center by NASA and The Catholic University of America in cooperation with the Naval Research Laboratory, and of the Radio Monitoring web site at LESIA UMR 8109, Observatoire de Paris, supported by the French Space Agency CNES. The authors acknowledge helpful discussions during the CESRA workshop 2019 in Potsdam. They are grateful to the referee for helpful comments, and to Eoin Carley for explaining some subleties of his mother tongue. CSM gratefully acknowledges Eric Sanchez for his support in handling the Blender tool, and Paris Observatory for a one-month stay in 2019. Research at Paris Observatory was supported by the Programme National Soleil-Terre (PNST) of CNRS/INSU.

\section{References}

Alissandrakis, C. E., Bouratzis, C., \& Hillaris, A. 2019, A\&A, 627, A133

Andrews, M. D. 2001, Sol. Phys., 204, 179

Aschwanden, M. J., \& Alexander, D. 2001, Sol. Phys., 204, 91

Bentley, R. D., Klein, K. L., van Driel-Gesztelyi, L., et al. 2000, Sol. Phys., 193 227

Benz, A. O., \& Wentzel, D. G. 1981, A\&A, 94, 100

Boischot, A. 1957, Acad. Sci. Paris C. R., 244, 1326

Bougeret, J.-L., Kaiser, M. L., Kellogg, P. J., et al. 1995, Space Sci. Rev., 71, 231

Brown, J. C. 1972, Sol. Phys., 26, 441

Brueckner, G. E., Howard, R. A., Koomen, M. J., et al. 1995, Sol. Phys., 162, 357

Carmichael, H. 1964, NASA Spec. Publ., 50, 451

Caroubalos, C., Alissandrakis, C. E., Hillaris, A., et al. 2001a, Sol. Phys., 204, 165

Caroubalos, C., Maroulis, D., Patavalis, N., et al. 2001b, Exp. Astron., 11, 23

Chertok, I. M., \& Grechnev, V. V. 2005, Sol. Phys., 229, 95

Delaboudinière, J.-P., Artzner, G. E., Brunaud, J., et al. 1995, Sol. Phys., 162, 291

Del Zanna, G., Aulanier, G., Klein, K., \& Török, T. 2011, A\&A, 526, A137

Démoulin, P., \& Aulanier, G. 2010, ApJ, 718, 1388

Dulk, G. A., \& Nelson, G. J. 1973, PASA, 2, 211

Elgarøy, E. Ø. 1977, Solar Noise Storms (Oxford: Pergamon Press)

Gary, D. E., Dulk, G. A., House, L. L., Illing, R., \& Wagner, W. J. 1985, A\&A, 152,42

Gergely, T. E., Kundu, M. R., Munro, R. H., \& Poland, A. I. 1979, ApJ, 230, 575

Habbal, S. R., Ellman, N. E., \& Gonzalez, R. 1989, ApJ, 342, 594

Hillaris, A., Bouratzis, C., \& Nindos, A. 2016, Sol. Phys., 291, 2049

Hirayama, T. 1974, Sol. Phys., 34, 323

Howard, R. A., Moses, J. D., Vourlidas, A., et al. 2008, Space Sci. Rev., 136, 67
Howard, T. A., DeForest, C. E., Schneck, U. G., \& Alden, C. R. 2017, ApJ, 834, 86

Huang, J., Démoulin, P., Pick, M., et al. 2011, ApJ, 729, 107

Hudson, H. S., Acton, L. W., \& Freeland, S. L. 1996, ApJ, 470, 629

Janvier, M., Aulanier, G., \& Démoulin, P. 2015, Sol. Phys., 290, 3425

Kahler, S. W., \& Hundhausen, A. J. 1992, J. Geophys. Res., 97, 1619

Kai, K., Melrose, D. B., \& Suzuki, S. 1985, in Solar Radiophysics: Studies of Emission from the Sun at Metre Wavelengths, eds. D. McLean, \& N. Labrum (Cambridge, Great Britain: Cambridge University Press), 415

Kerdraon, A., \& Delouis, J. M. 1997, in Coronal Physics from Radio and Space Observations, ed. G. Trottet (Berlin: Springer Verlag), Lect. Notes Phys., 483, 192

Kerdraon, A., Pick, M., Hoang, S., Wang, Y., \& Haggerty, D. 2010, ApJ, 715, 468

Klein, K.-L., \& Mouradian, Z. 2002, A\&A, 381, 683

Klein, K.-L., Trottet, G., Lantos, P., \& Delaboudinière, J.-P. 2001, A\&A, 373, 1073

Kopp, R. A., \& Pneuman, G. W. 1976, Sol. Phys., 50, 85

Koval, A., Stanislavsky, A., Chen, Y., et al. 2016, ApJ, 826, 125

Kraus, J. D. 1966, Radio Astronomy (New York: McGraw-Hill)

Lantos, P., Kerdraon, A., Rapley, G. G., \& Bentley, R. D. 1981, A\&A, 101, 33

Lecacheux, A. 2000, Wash. DC Am. Geophys. Union Geophys. Monogr. Ser., 119,321

Lemen, J. R., Title, A. M., Akin, D. J., et al. 2012, Sol. Phys., 275, 17

Liu, H., Chen, Y., Cho, K., et al. 2018, Sol. Phys., 293, 58

MacQueen, R. M. 1980, R. Soc. London Philos. Trans. Ser., 297, 605

Maia, D., Pick, M., Hawkins, S. E., III, Fomichev, V. V., \& Jiřička, K. 2001, Sol. Phys., 204, 197

Masson, S., Antiochos, S. K., \& DeVore, C. R. 2019, ApJ, 884, 143

McCauley, P. I., Cairns, I. H., White, S. M., et al. 2019, Sol. Phys., 294, 106

Melrose, D. B. 1980, Sol. Phys., 67, 357

Melrose, D. B. 2017, Rev. Mod. Plasma Phys., 1, 5

Morosan, D. E., Zucca, P., Bloomfield, D. S., \& Gallagher, P. T. 2016, A\&A, 589, L8

Morosan, D. E., Kilpua, E. K. J., Carley, E. P., \& Monstein, C. 2019, A\&A, 623, A63

Morosan, D. E., Palmerio, E., Pomoell, J., et al. 2020, A\&A, 635, A62

Pick, M. 1986, Sol. Phys., 104, 19

Pick, M., \& Vilmer, N. 2008, A\&ARv, 16, 1

Pick-Gutmann, M. 1961, Annal. Astrophys., 24, 181

Raulin, J. P., \& Klein, K.-L. 1994, A\&A, 281, 536

Régnier, S. 2015, A\&A, 581, A9

Riddle, A. C. 1970, Sol. Phys., 13, 448

Robinson, R. D. 1978, Aust. J. Phys., 31, 533

Robinson, R. D. 1985, in Solar Radiophysics: Studies of Emission from the Sun at Metre Wavelengths, eds. D. McLean, \& N. Labrum (Cambridge, Great Britain: Cambridge University Press), 385

Robinson, R. D. 1986, Sol. Phys., 104, 33

Salas-Matamoros, C., Klein, K.-L., \& Rouillard, A. P. 2016, A\&A, 590, A135

Scherrer, P. H., Bogart, R. S., Bush, R. I., et al. 1995, Sol. Phys., 162, 129

Schrijver, C. J., \& De Rosa, M. L. 2003, Sol. Phys., 212, 165

Seaton, D. B., Berghmans, D., Nicula, B., et al. 2013, Sol. Phys., 286, 43

Sharma, R. R., \& Vlahos, L. 1984, ApJ, 280, 405

Song, H. Q., Zhang, J., Li, L. P., et al. 2019, ApJ, 887, 124

Spicer, D. S., Benz, A. O., \& Huba, J. D. 1982, A\&A, 105, 221

Stewart, R. T. 1985, in Solar Radiophysics: Studies of Emission from the Sun at Metre Wavelengths, eds. D. McLean, \& N. Labrum (Cambridge, Great Britain: Cambridge University Press), 361

Stupp, A. 2000, MNRAS, 311, 251

Sturrock, P. A. 1966, Nature, 211, 695

Török, T., Downs, C., Linker, J. A., et al. 2018, ApJ, 856, 75

Vlahos, L., Gergely, T. E., \& Papadopoulos, K. 1982, ApJ, 258, 812

Wang, J.-X., Zhou, G.-P., Wen, Y.-Y., et al. 2006, Chin. J. Astron. Astrophys., 6, 247

Webb, D. F., \& Howard, T. A. 2012, Liv. Rev. Sol. Phys., 9, 3

Weiss, A. A. 1963, Aust. J. Phys., 16, 526

Wentzel, D. G. 1985, ApJ, 296, 278

Wentzel, D. G. 1986, Sol. Phys., 103, 141

Wentzel, D. G., Zlobec, P., \& Messerotti, M. 1986, A\&A, 159, 40

Wild, J. P. 1969, Sol. Phys., 9, 260

Wild, J. P. 1970, PASA, 1, 365

Wild, J. P., Smerd, S. F., \& Weiss, A. A. 1963, ARA\&A, 1, 291

Wuelser, J. P., Lemen, J. R., Tarbell, T. D., et al. 2004, in Telescopes and Instrumentation for Solar Astrophysics, eds. S. Fineschi, \& M. A. Gummin, Proc. SPIE, 5171, 111

Yan, Y., Aschwanden, M. J., Wang, S., \& Deng, Y. 2001, Sol. Phys., 204, 27

Zhang, H. 2002, MNRAS, 332, 500

Zhukov, A. N., \& Auchère, F. 2004, A\&A, 427, 705 


\section{Appendix A: October 9, 2001}

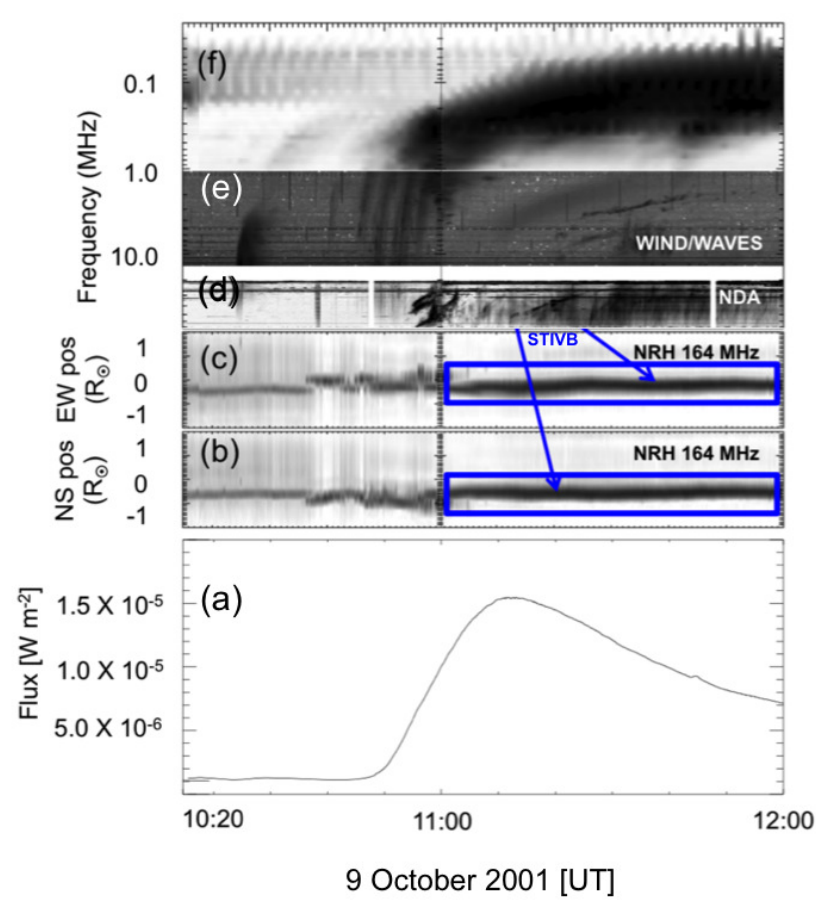

Fig. A.1. Multifrequency plot of soft X-ray and radio emissions on October 9, 2001. Panels show: $(a)$ soft X-ray profile; $(b)$ 1D images projected on the solar north-south and $(c)$ east-west directions ( $y$ axis graded in solar radii) at $164 \mathrm{MHz}$; the stationary type IV burst (STIVB) is enclosed in the blue rectangles. $(d)-(f)$ : dynamic spectra between $80 \mathrm{MHz}$ and $10 \mathrm{kHz}$.

At 11:30 UT on October 9, 2001 a halo CME with speed $\sim 1000 \mathrm{~km} \mathrm{~s}^{-1}$ was observed by SoHO/LASCO. This CME was associated with a filament eruption and a soft X-ray burst as shown in the bottom panel of Fig. A.1. The onset and the peak of the soft X-ray burst were observed at $\sim 10: 46$ UT and $\sim 11: 13$ UT, respectively. The sequence of $1 \mathrm{D}$ images taken by the NRH at $164 \mathrm{MHz}$ (Figs. A.1b, c) shows a noise storm before 10:30 UT, followed by a complex containing moving type IV sources, and then the stationary type IV burst starting near 11:00 UT (enclosed in the blue rectangles). The stationary type IV emission was observed at all NRH frequencies (432, 411, 327, 237 and $164 \mathrm{MHz}$ ). The spectrum observed by the Nançay Decameter Array (NDA; Lecacheux 2000) is displayed in Fig. A.1c. It extended down to $30 \mathrm{MHz}$. In this range the stationary type IV burst was preceded by a type II burst around 11:00 UT. Since the NDA antennas have reduced sensitivity at lower frequencies, the actual cutoff may still be lower. But it seems to be above $14 \mathrm{MHz}$, since no counterpart is observed by Wind/WAVES (Figs. A.1e, f).

\section{A.1. Geometry of the radio source and the magnetic field}

Figure A.2 shows coronal magnetic field lines extrapolated with the PFSS model from the photospheric magnetogram observed by SoHO/MDI at 12:04, overlaid on the magnetogram (a) and an EUV image $(19.5 \mathrm{~nm}$; b, c). Contours of the stationary type IV source at different frequencies are superposed in Fig. A.2b. All radio sources are located in the eastern branch of a large-scale coronal structure (a loop system in the PFSS extrapolation). They form an overall source with columnar structure. Its buildup was observed between 10:53 UT (at $432 \mathrm{MHz}$ ) and 10:57 UT (at
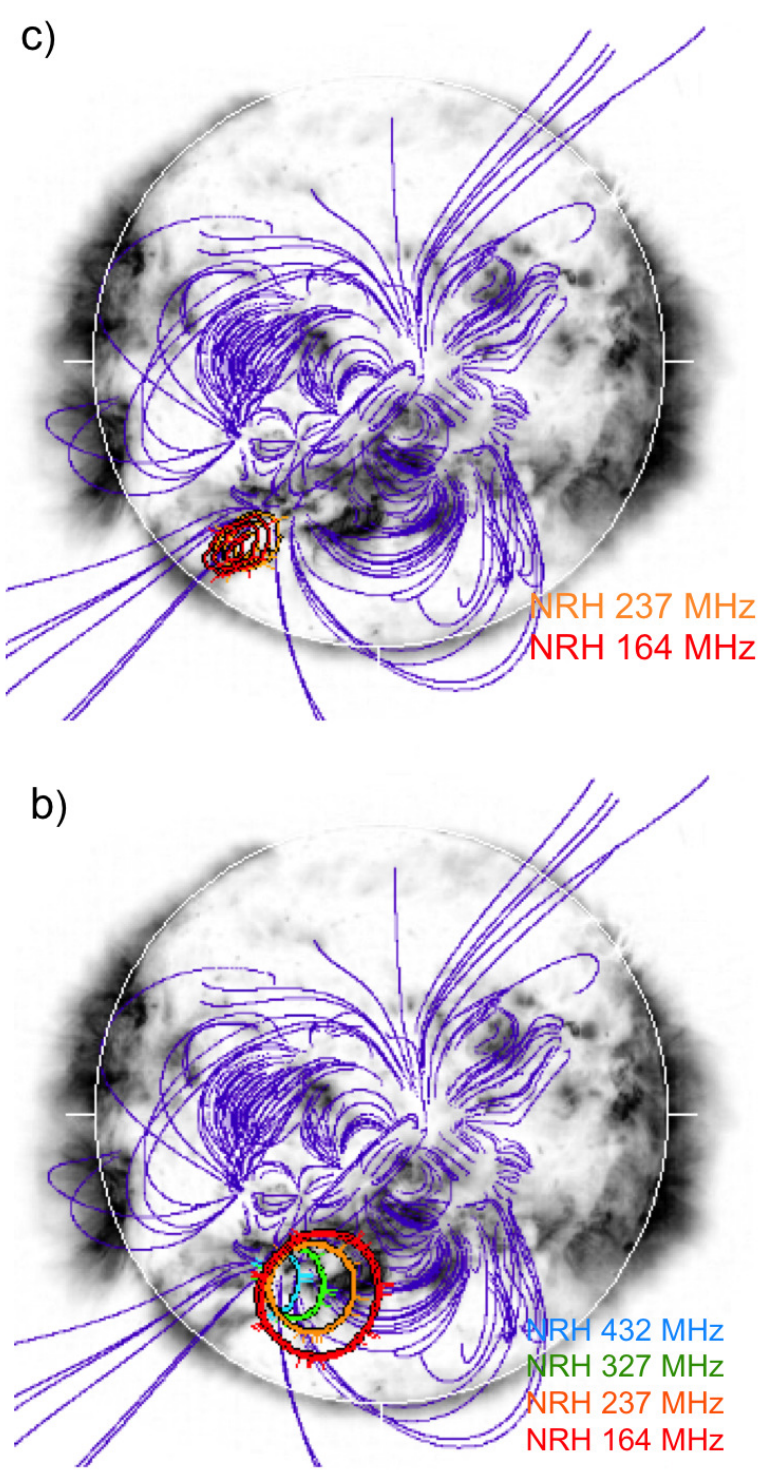

a)

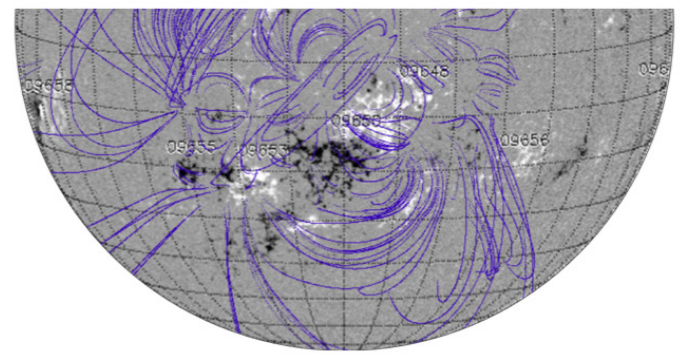

Fig. A.2. Superposition of the magnetic field lines from the PFSS model on an MDI magnetogram $(a)$ and an EIT $19.5 \mathrm{~nm}$ image $(b-c)$ on October 9, 2001. Radio sources of the stationary type IV continuum seen in Stokes parameter $I$ at different frequencies are plotted in $(b)$, and a noise storm in $(c)$. Contours of equal brightness temperature at $50 \%$ of the maximum are plotted in colours to represent different frequencies.

$164 \mathrm{MHz}$ ) before the peak of the flare. SoHO/EIT images show the appearance of flare ribbons between 10:48 and 11:00 UT. At 11:12 they are connected by an arcade of loops. Within the limits of the 12 min cadence of EIT, we can hence conclude that the stationary type IV burst appeared near the times when flare ribbons and the arcade of loops that connects them were established. 

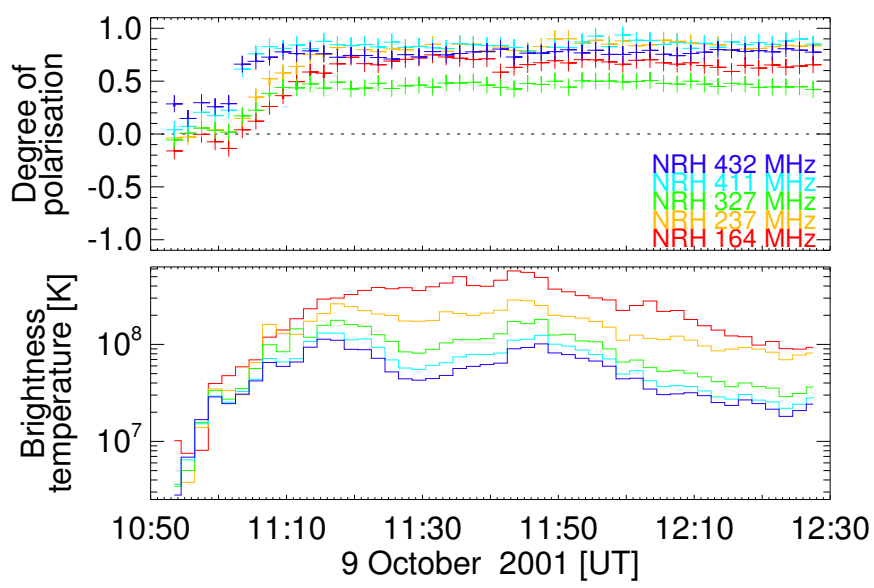

Fig. A.3. Polarisation degree and brightness temperature profiles for the stationary type IV source on October 9, 2001. Integration time 2 min.

The radio sources at high frequencies project onto the eastern part of the flare-loop arcade seen in EUV. The sources at lower frequencies are located more to the west, suggesting that the magnetic structure was curved westward, towards the leading part of the parent active region (see Fig. A.2b), just as the extrapolated coronal magnetic field lines. This configuration prevailed during $1-1.5 \mathrm{~h}$. Thereafter the multifrequency sources were aligned close to the line of sight. This evolution could indicate the outward expansion of the loop structure. Movements of small amplitude were observed, but they were likely due to variations of the refractive index in the ionosphere.

\section{A.2. Circular polarisation}

The stationary type IV emission was left-hand polarised $(V>0)$ at all frequencies. Figure A.2a shows that the field lines on which the radio sources project are rooted in a region of positive magnetic polarity in active region 9653 . This implies polarisation in the ordinary mode. The time history of the polarisation degree during the first $1.5 \mathrm{~h}$ is plotted in Fig. A.3 (top panel). The profile shows an increase in the polarisation degree during the rise phase of the soft X-ray burst to a level of $40 \%$ to $80 \%$, depending on frequency. The polarisation remained at this high level after 12:30 UT, while the brightness temperature (bottom panel) of the stationary type IV source gradually faded. The averages of the degree of circular polarisation and the logarithm of the brightness temperature in Cols. 8, 9 of Table 1 were computed in the interval 11:20-12:30 UT.

To study the rise of the polarisation degree in more detail, we identified visually the times when a clearly polarised source became visible for the first time, and when the degree of polarisation ceased to increase. During this interval the brightness temperature, the degrees of polarisation at the brightest points in the maps in Stokes parameters $I$ and $V$, and the distance between these points were calculated. Figure A.4 shows scatter plots of the brightness temperature (left panels) and the distance (right panels) vs degree of polarisation at the two lowest observed frequencies. The degree of polarisation in the brightest pixel of the $I$-map is plotted by a plus, that in the brightest pixel of the $V$-map by a diamond. The two values are different whenever the brightest pixels in the $I$ and $V$-maps differ. Data with 2 min integration are used, and the field of view of 4 solar radii corresponds to 256 pixels. The left panels in Fig. A.4 show that the polarisation increases as the peak brightness temperature increases. Right panels show the trend that when the polarisation is low, the
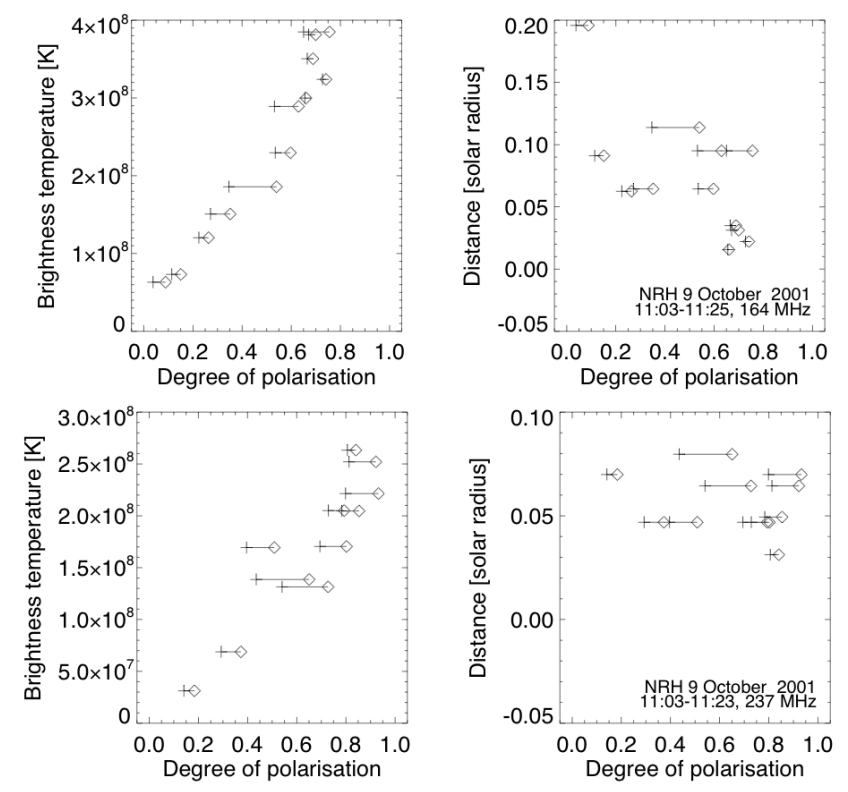

Fig. A.4. Scatter plots of the brightness temperature (left panels) and the distance between the brightest pixels in the $I$ and $V$-maps versus degree of circular polarisation, during the phase of rising polarisation on October 9, 2001. Integration time $2 \mathrm{~min}$.

peak positions in the $I$ and $V$-maps are different, but that the two positions get closer and merge as the polarisation increases. The trend is clearer at $164 \mathrm{MHz}$ than at the higher frequencies, where some scatter remains throughout the rise phase. These plots show that the apparently simple source in Stokes parameter $I$ had unresolved fine structure with different degrees of polarisation, with a trend that emission becomes more and more concentrated into one single source as the degree of polarisation increases. This evolution suggests that the strongly polarised source emerged out of a set of unresolved sources with different degrees of polarisation, and that the degree of polarisation increased because the strongly polarised components gradually became dominant.

\section{A.3. Noise storm}

As on July 14, 2000, a noise storm was observed before the flare, and it was close to the subsequent stationary type IV location. Figure A.2c shows the superposition of the noise storm sources seen in Stokes parameter $I$ for the two lowest frequencies on SoHO/EIT images, combined with the PFSS-extrapolated magnetic field configuration. Quiet Sun emission was observed at the higher frequencies.

The noise storm was strongly polarised $(>80 \%$ at $164 \mathrm{MHz}$, $100 \%$ at $237 \mathrm{MHz})$ in the right-hand sense $(V<0)$, i.e. opposite to the stationary type IV continuum. It also presented a columnar structure, which was oriented south-eastwards, hence away from the stationary type IV source. This is consistent with its location in the magnetic field configuration in Fig. A.2. Field lines through the noise storm sources connect the high corona with a region of negative photospheric polarity to the east of the roots of the field lines through the stationary type IV source. The right-hand polarisation of the noise storm is hence consistent with the ordinary mode. We note for later discussion that the polarisation of the noise storm was stronger than that of the stationary type IV burst. This means that the partial polarisation of the stationary type IV source is not an instrumental artefact. 


\section{Appendix B: May 19, 2007}

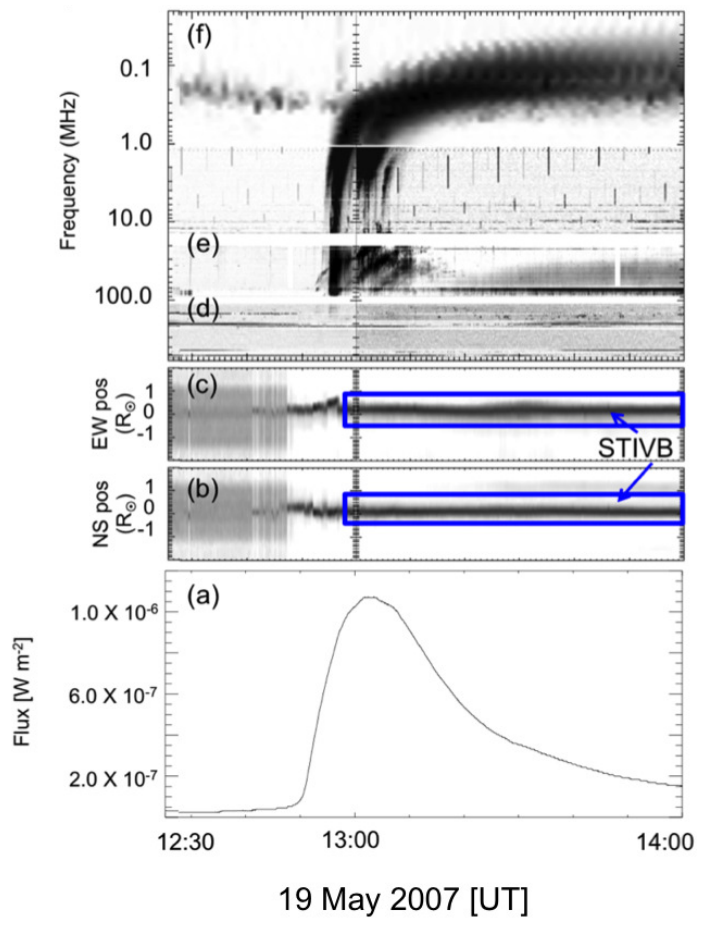

Fig. B.1. Multifrequency plot of soft X-ray and radio emissions on May 19, 2007. Panels show: $(a)$ soft X-ray profile; $(b)$ 1D images projected on the solar north-south and $(c)$ east-west directions $(y$ axis graded in solar radii) at $150 \mathrm{MHz}$; the stationary type IV burst (STIVB) is enclosed by the blue rectangles. $(d)-(f)$ : dynamic spectra between $650 \mathrm{MHz}$ and $10 \mathrm{kHz}$.

SoHO/LASCO observed a CME of width $\sim 100^{\circ}$ and speed $960 \mathrm{~km} \mathrm{~s}^{-1}$, with the first image in C2 at 13:24 UT. The associated soft X-ray burst onset and peak were detected by GOES at 12:50 UT and 13:02 UT, respectively, as shown in Fig. B.1a. The initial development of the flare loops was observed at the western edge of the active region by STEREO/EUVI and the $\mathrm{H}_{\alpha}$ telescope at Kanzelhöhe Observatory ${ }^{4}$ between 12:49 UT and 12:52 UT. Figure B.1 presents a multifrequency compilation of the event. The 1D NRH images at $150 \mathrm{MHz}$ (Figs. B.1b, c) show quiet sun emission and a faint noise storm until the onset of the soft X-ray burst, a complex with moving sources until about 12:57 UT, and a stationary type IV burst thereafter (enclosed within blue rectangles). The stationary type IV burst was observed by NDA, preceded by a type II burst. The low-frequency limit of the stationary type IV spectrum drifted gradually down to $30 \mathrm{MHz}$. No counterpart was observed by Wind/WAVES. The low-frequency cutoff of the stationary type IV spectrum was hence between 30 and $14 \mathrm{MHz}$. Kerdraon et al. (2010) conducted a detailed analysis of the event before the stationary type IV burst.

The sources of the stationary type IV continuum at different frequencies showed a columnar structure, which built up between 12:49 UT (at $432 \mathrm{MHz}$ ) and 13:03 UT (at $150 \mathrm{MHz}$ ). It was located above the only active region on the solar disk, NOAA 10956. As shown in the SoHO/MDI magnetogram (Fig. B.2a), the active region had a large region of negative (southward) polarity in its centre, with opposite polarities at its south-western and south-eastern edges. The radio sources at dif-

\footnotetext{
4 https://www.kso.ac.at/index.php
}
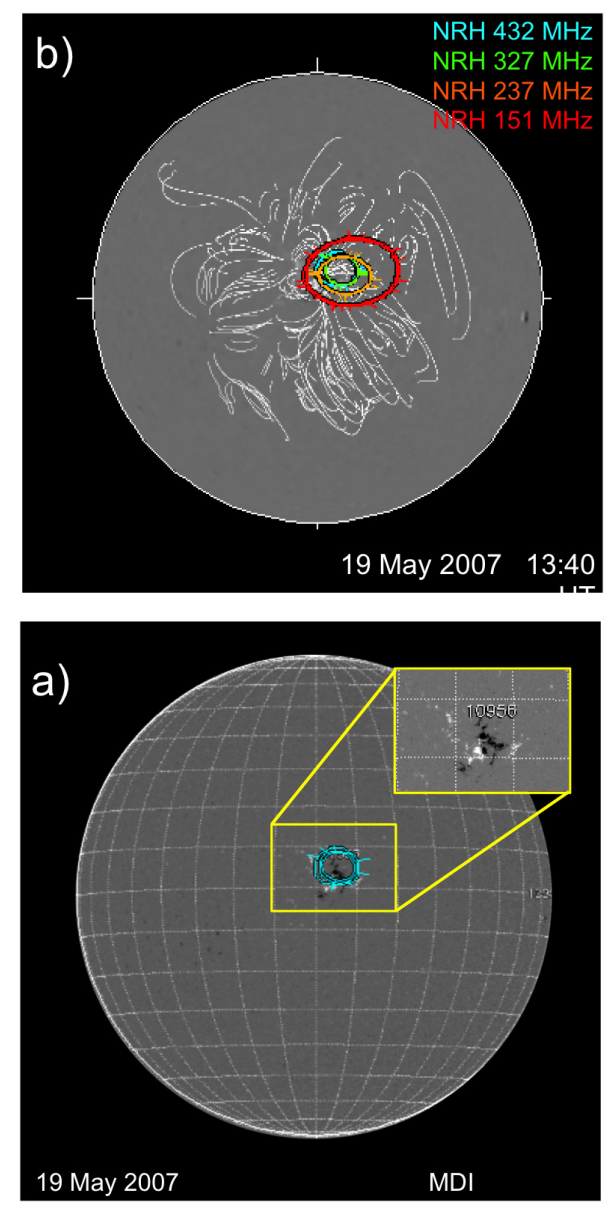

Fig. B.2. $a$ : MDI magnetogram showing the magnetic polarities of the active region NOAA 10965 on May 19, 2007 with susperposed isointensity contours at half maximum of the stationary type IV continuum at $432 \mathrm{MHz}$. $b$ : superposition of radio sources seen in Stokes parameter $I$ on the magnetic field configuration inferred by the PFSS model of Schrijver \& De Rosa (2003). The contours of equal brightness temperature at $50 \%$ of the maximum represent different frequencies of the NRH.

ferent frequencies were aligned along the line of sight. It projects onto the region of southward magnetic polarity, where the loopshaped field lines inferred from the PFSS extrapolation converge (Fig. B.2b).The circular polarisation was right-handed $(V<0)$ at all frequencies. This suggests polarisation in the ordinary mode.

The time profile of the polarisation degree is shown in the top panel of Fig. B.3. The polarisation degree increased regularly during the first $30 \mathrm{~min}$ of the event, and then stayed constant on a high level $(60 \%-100 \%)$. At frequencies above $300 \mathrm{MHz}$ the stationary type IV continuum was at times so weak that the thermal corona made a significant contribution to the total intensity. The thermal contribution to $I$ was subtracted as described in Sect. 2.3. The observations at the lower frequencies are consistent with full polarisation, while the polarisation is found to be high, but partial, above $400 \mathrm{MHz}$. The average values of the degree of circular polarisation and the logarithm of the brightness temperature in Cols. 8, 9 of Table 1 were computed in the interval 13:30-14:30 UT.

The time profile of the brightness temperature has two different parts: a relatively bright initial one with a drop at the higher frequencies (327-432 MHz) near 13:12 UT, followed by a second part where the brightness temperature decayed slowly at high frequencies and stayed essentially constant at the low 


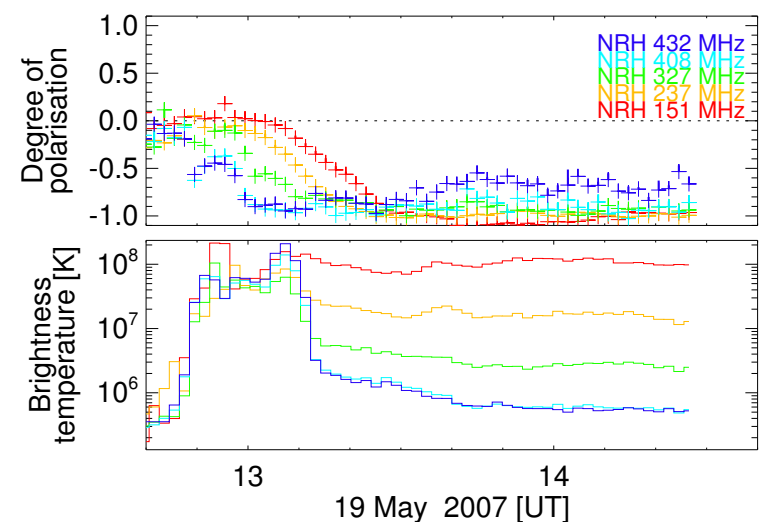

Fig. B.3. Polarisation degree and brightness temperature profiles on May 19, 2007. Integration time $2 \mathrm{~min}$.
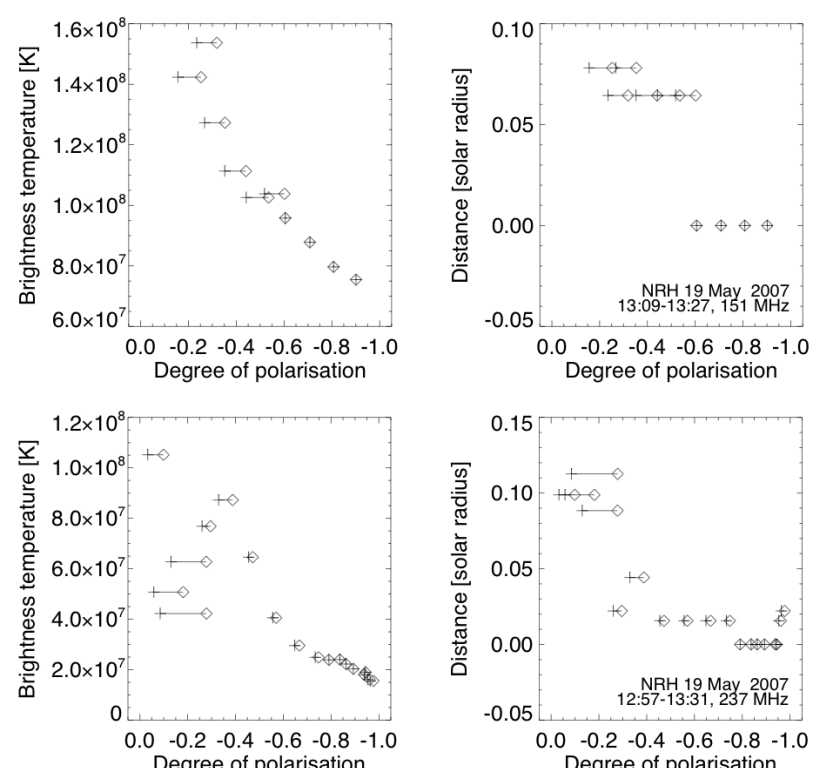

Fig. B.4. Scatter plots of the brightness temperature (left panels) and the distance between the brightest pixels in the $I$ and $V$-maps versus degree of circular polarisation, during the phase of rising polarisation on May 19, 2007. Integration time $2 \mathrm{~min}$.

frequencies. The increase of the polarisation started during the bright initial part at the high frequencies, and near its end at the low frequencies. Its time evolution is clearly different from that of the brightness temperature. Scatter plots in Fig. B.4 show that, as in the October 9th event, the peak positions in the $I$ and $V$-maps converge as the polarisation increases, again revealing signatures of unresolved source structure during the rise of the polarisation degree.

\section{Appendix C: April 26, 2008}

The event on April 26, 2008 has been studied in detail by Huang et al. (2011) and Salas-Matamoros et al. (2016). SoHO/LASCO observed a CME with speed $515 \mathrm{~km} \mathrm{~s}^{-1}$ and width $280^{\circ}$, with the first image in C2 at 14:30 UT. It was associated with a flare whose soft X-ray emission started at 13:50 UT and peaked at 14:08 UT (see Fig. C.1a). The initial steps in the time profile of the $0.1-0.8 \mathrm{~nm}$ channel are artefacts of the A/D conversion. Figures C.1b, c show 1D images at $327 \mathrm{MHz}$ and $150 \mathrm{MHz}$, respectively, in the east-west direction. Only quiet sun
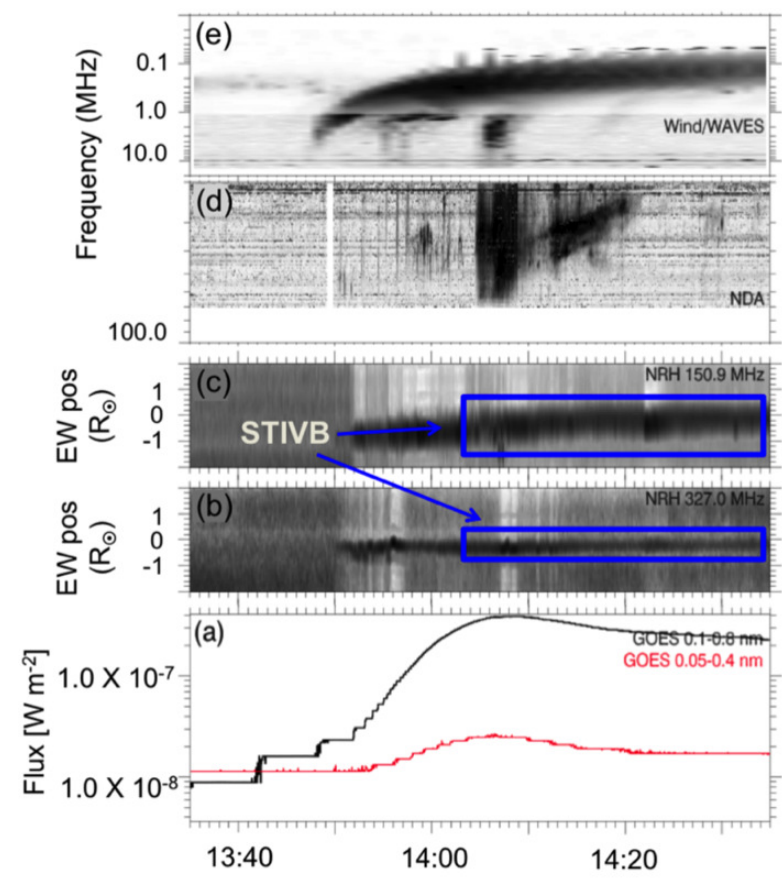

26 April 2008 [UT]

Fig. C.1. Multifrequency plot of soft X-ray and radio emissions. Panels show: $(a)$ soft X-ray profile; $(b)$ 1D images projected on the solar eastwest direction at $327 \mathrm{MHz}(b)$ and $150 \mathrm{MHz}(c)$; $(y$ axis graded in solar radii); the stationary type IV burst (STIVB) is enclosed in the blue rectangles. $d-e$ : dynamic spectra between $80 \mathrm{MHz}$ and $10 \mathrm{kHz}$. Plot from Salas-Matamoros et al. (2016).

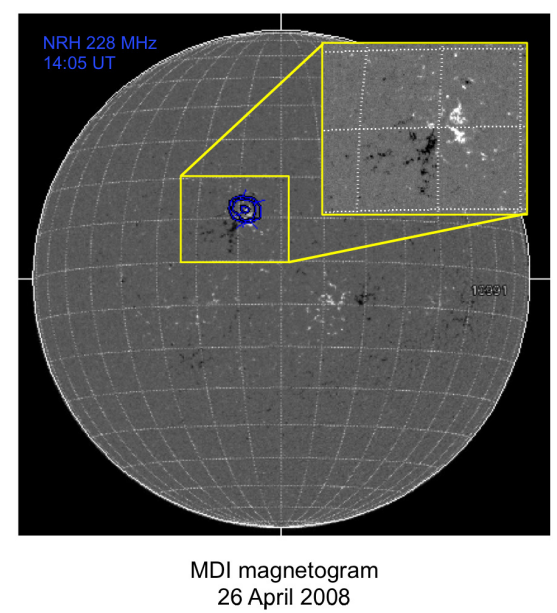

Fig. C.2. Superposition of radio sources seen in Stokes parameter $I$ on an EIT $19.5 \mathrm{~nm}$ image and of the source at $228 \mathrm{MHz}$ on an MDI magnetogram for the stationary type IV source on April 26, 2008.

emission is seen at both frequencies before the soft X-ray burst. The radio emission started with a westward moving source during the rise of the soft X-ray burst, followed by the stationary type IV burst (inside the blue rectangles). No signature of the stationary type IV continuum was detected below $80 \mathrm{MHz}$ by either NDA or Wind/WAVES.

The stationary type IV source was often seen on top of a significant thermal background. The sources formed a columnar structure along the line of sight above the active region (Fig. C.2a). The axis was slightly directed towards the eastern 
hemisphere in the first tens of minutes, and then gradually turned westwards. The sources at $150 \mathrm{MHz}$ and $228 \mathrm{MHz}$ show at times evidence of unresolved or partially resolved structure. The source in Stokes parameter $I$ at $228 \mathrm{MHz}$, which is representative of the radio sources at all frequencies, is overplotted onto an MDI magnetogram in Fig. C.2b. The source is seen to project onto the region with positive polarity. Between 14:22 UT and 14:23 UT a second source, also with columnar structure, briefly appeared above the region of southern polarity in the photosphere.

The sources at 150,228 and $327 \mathrm{MHz}$ were left-hand polarised $(V>0)$, while the short-lasting source above the opposite photospheric polarity near 14:23 UT was right-hand polarised. The radio emission is hence in the ordinary mode. The emission at $150 \mathrm{MHz}$ displayed a systematic increase of the degree of polarisation from about 20\% (near 14:02 UT) to above $80 \%$ between 14:20 UT and the end of the NRH observations. An increase of the polarisation was also observed at 228 and $327 \mathrm{MHz}$, but the degree was lower. At 408 and $432 \mathrm{MHz}$ no significant circular polarisation was seen after 13:58 UT, although the polarisation was high just before, and the source was clearly defined in $I$ until the end of the NRH observing time. Hence, the stationary type IV emission was unpolarised at 408 and $432 \mathrm{MHz}$. The average values of the degree of circular polarisation and the logarithm of the brightness temperature in Cols. 8, 9 of Table 1 were computed in the interval 14:20-15:10 UT.

The stationary type IV burst appeared in a broad window together with an arcade of EUV loops, which existed in the EIT $19.5 \mathrm{~nm}$ image at 14:00 UT, but not at 13:48 UT. The radio sources project onto the arcade of loops, rather to its western end where a dimming region was observed. However, the localisation is less clear than in other cases, such as July 14, 2000 and October 9, 2001.

\section{Appendix D: April 3, 2010}

SoHO/LASCO observed a halo CME with a speed of $670 \mathrm{~km} \mathrm{~s}^{-1}$. It was first seen in LASCO/C2 at 10:34 UT, and accompanied by a soft X-ray burst with onset at 8:50 and peak at 10:00 UT (see Fig. D.1a) in active region NOAA 11059 (S22 $\mathrm{W} 29^{\circ}$ ). The soft X-ray profile had a complex rise, and the first appearance of flare loops was difficult to identify. Inspection of the EIT movie at $19.5 \mathrm{~nm}$ suggests that loops clearly bridging the principal inversion line of the photospheric magnetic field appeared between 09:48 and 10:00 UT, while the interval inferred from the Proba2/SWAP daily movie at $17.1 \mathrm{~nm}$ was later, 09:54-10:14 UT.

The 1D NRH images (Figs. D.1b and c) show quiet sun emission before the flare onset. Then an intense radio source was observed, which moved to the western limb during the impulsive phase of the flare. The dynamic spectrum between 20 and $80 \mathrm{MHz}$ (Fig. D.1e) shows the low-frequency extension of this moving type IV burst as a faint drifting feature after the soft $\mathrm{X}$-ray burst peak. This moving type IV burst was accompanied by a type III burst in the interplanetary space (Fig. D.1f). The long lasting source observed at $150 \mathrm{MHz}$ after about 10:00 UT is the stationary type IV source (enclosed by blue rectangles in Figs. D.1b, c). It was not observed at frequencies below $80 \mathrm{MHz}$ by NDA or Wind/WAVES. The source of the stationary type IV continuum appeared first at 9:50 UT at $327 \mathrm{MHz}$, but preexisting sources hid the onset at lower frequencies.

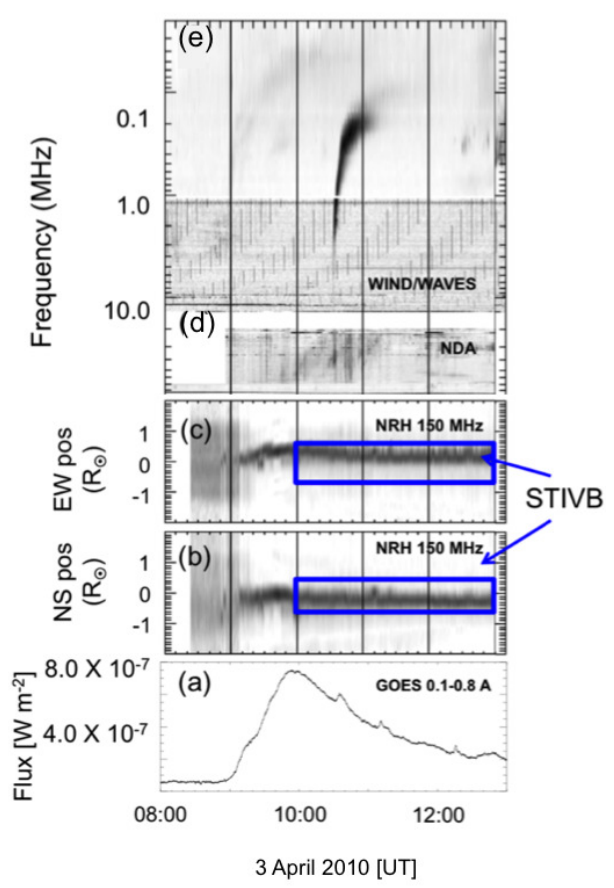

Fig. D.1. Multifrequency plot of soft X-ray and radio emissions. Panels show: $(a)$ soft X-ray profile; $(b)$ 1D images projected on the solar north-south and (c) east-west directions ( $y$ axis graded in solar radii) at $150 \mathrm{MHz}$; the stationary type IV burst (STIVB) is enclosed by the blue rectangles. $(d)-(e)$ : dynamic spectra between $100 \mathrm{MHz}$ and $10 \mathrm{kHz}$.
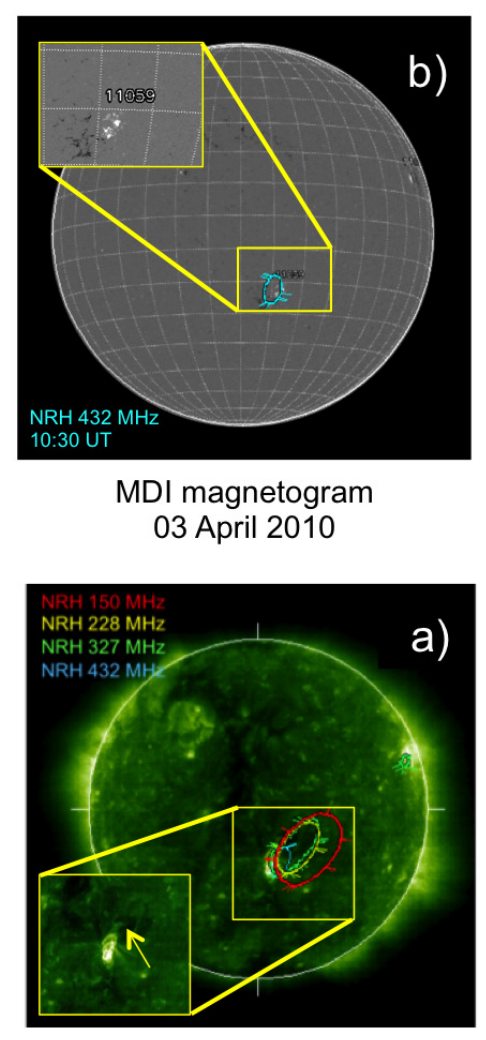

EIT 195

03 April 2010 10:30 UT

Fig. D.2. Superposition of radio sources seen in Stokes parameter $I$ on an EIT $19.5 \mathrm{~nm}$ image (a) and a MDI magnetogram $(b)$ for the stationary type IV source on April 3, 2010. The contours of equal brightness temperature at $50 \%$ of the maximum are plotted in colours to represent different frequencies. 


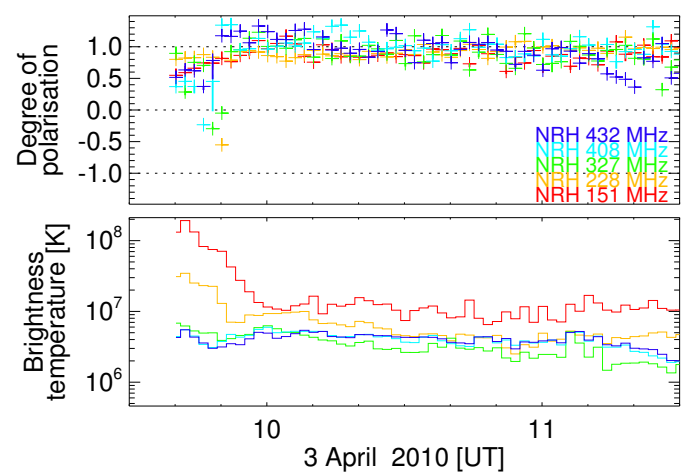

Fig. D.3. Polarisation degree and brightness temperature profiles for the stationary type IV source on April 3, 2010. Integration time 2 min.

The $50 \%$ iso-intensity contours of the stationary type IV sources are overlaid onto the EIT image at $19.5 \mathrm{~nm}$ in Fig. D.2a. The source presented most of the time a columnar structure located at the western side of the flaring active region, at the northern end of an arcade of flare loops that extended towards south. At this location, a dimming region was also observed in EUV images, as indicated by the yellow arrow in Fig. D.2a. On occasion the source appeared elongated or with two components at frequencies below $300 \mathrm{MHz}$. As seen in the overlay on the photospheric magnetogram in Fig. D.2b, the radio source was located above the positive polarity area of the active region. Since the polarisation of the stationary type IV source was found to be left-handed, this location implies emission in the ordinary mode.

The time histories of the degree of polarisation in Fig. D.3 show that during the entire stationary type IV continuum the source was fully polarised. Indications of a rise before 10:00 UT probably belong to the type IV emission before the stationary continuum. They occurred while the brightness temperature decreased at the lower frequencies. The brightness temperature was about constant during the phase of strong polarisation. The average degree of circular polarisation and the logarithm of the brightness temperature in Cols. 8, 9 of Table 1 were computed in the interval 10:10-11:30 UT.

\section{Appendix E: March 4, 2012}

On March 4, 2012 a halo CME with speed $\sim 1300 \mathrm{~km} \mathrm{~s}^{-1}$ was observed by LASCO C2 at 11:00 UT, associated with a flare located in active region NOAA 11429 close to the eastern solar limb. The soft X-ray and radio emissions associated with this event are shown in Fig. E.1. The soft X-ray burst started at 10:29 UT and peaked at 10:52 UT (Fig. E.1a). Flare loops were first observed in EUV images by SDO AIA (19.3 nm) between 10:30 UT and 10:39 UT. 1D NRH images at $150 \mathrm{MHz}$ in the north-south and east-west directions in Figs. E.1b and c, show a noise storm before the soft X-ray burst followed by a moving type IV source above the eastern limb during the rise phase of the soft X-ray burst. The stationary type IV continuum appeared after 11:00 UT (enclosed in blue squares, Figs. E.1b, c).

The dynamic spectrum in the range $140-1000 \mathrm{MHz}$ observed by the ORFEES spectrograph at the Nançay radio observatory in Fig. E.1d shows two broadband emissions. The first, from 10:35 UT to 10:40 UT between 1000 and $300 \mathrm{MHz}$, has an onset drifting towards lower frequencies below about $300 \mathrm{MHz}$. This became the dominant emission below $80 \mathrm{MHz}$, as shown by the NDA spectrum in Fig. E.1e. Wind/WAVES

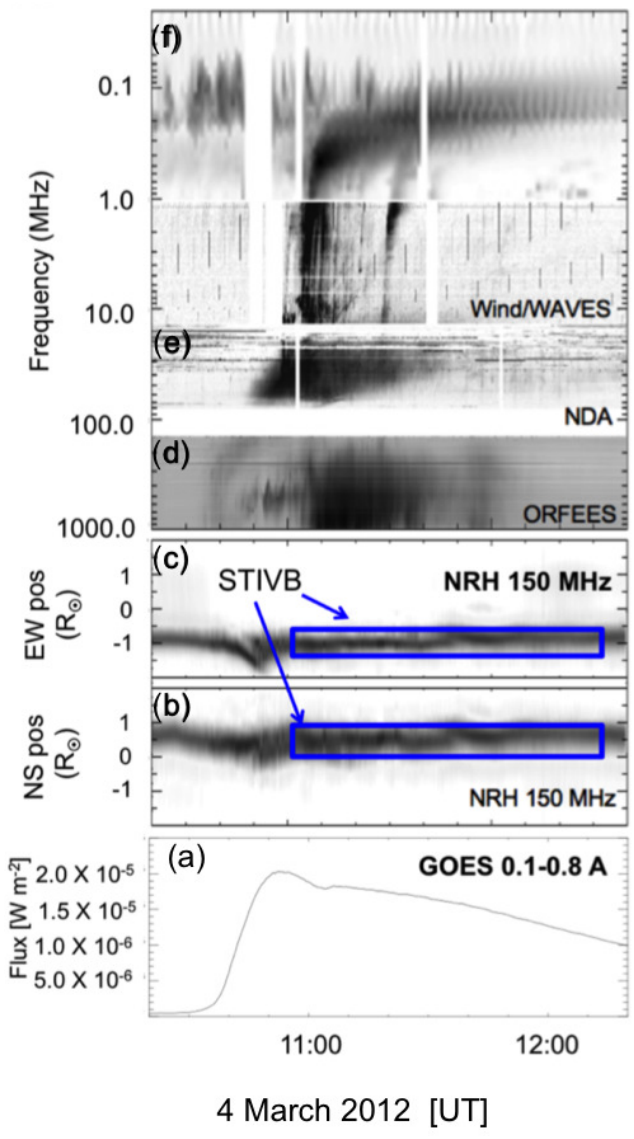

Fig. E.1. Multifrequency plot of soft X-ray and radio emissions. Panels show: $(a)$ soft X-ray profile; $(b)$ 1D images projected on the solar north-south and $(c)$ east-west directions ( $y$ axis graded in solar radii) at $150 \mathrm{MHz}$; the stationary type IV burst (STIVB) is enclosed by the blue rectangles. $(d)-(f)$ : dynamic spectra between $1000 \mathrm{MHz}$ and $10 \mathrm{kHz}$.

detected this continuum until 12:00 UT down to frequencies of about $8 \mathrm{MHz}$ (Hillaris et al. 2016). At $150 \mathrm{MHz}$ the drifting continuum is a moving type IV burst. This emission coincided with the rise of the soft X-ray burst. The second emission in the dynamic spectrum is an early manifestation of the stationary type IV source. It was observed at all NRH frequencies (150-445 MHz), but had no obvious counterpart in the NDA spectrum.

The NRH images show that the stationary type IV emission developed behind the rising moving type IV source. Two sources were seen during some time, on either side of the line connecting the moving type IV source to the centre of the solar disk. The southern source faded away after some minutes, while the northern one persisted until the end of the NRH observations near 15:00 UT. The northern source started the later, the lower the frequency, from 10:38 UT $(432 \mathrm{MHz})$ to $10: 50 \mathrm{UT}(150 \mathrm{MHz})$. The source seen in Stokes $I$ is broader than in $V$, which can be ascribed to unresolved fine structure.

Both the southern and the northern sources had columnar configuration, as seen in Stokes $V$ in Fig. E.3a and in Stokes $I$ in Fig. E.2b for the persistent northern source. The two sources were oppositely polarised, with left-handed sense in the persistent northern source. The polarisation was strong for some time, near $50 \%$ at several frequencies in the time interval 11:00-12:00 UT, for which the averages in Cols. 8, 9 of Table 1 were computed. But both the polarisation and the brightness temperature decreased after 12:00 UT. Since the active region was located close to the solar limb, the association between the 


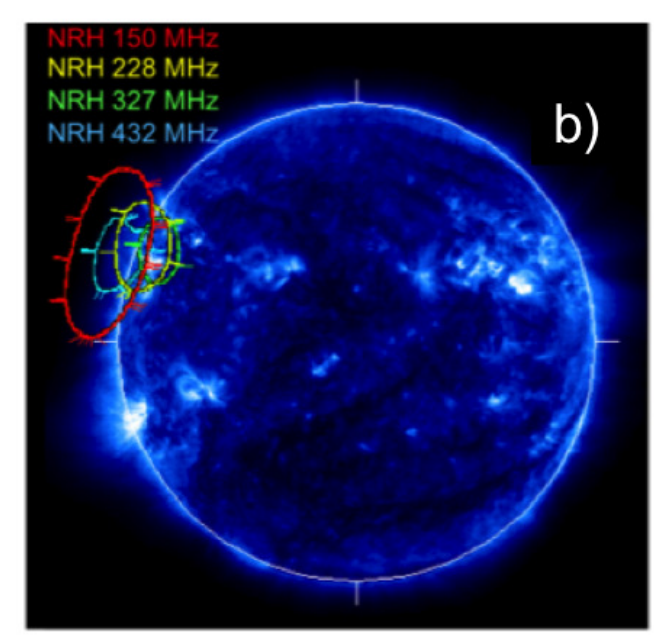

EIT 171

4 March 2012- 11:20 UT

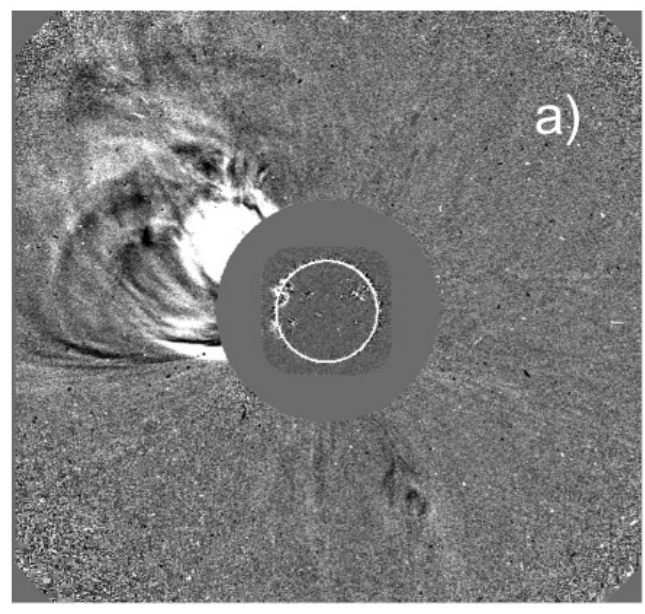

\section{LASCO C2 Dif}

4 March 2012- 11:00 UT

Fig. E.2. LASCO C2 difference image (a) and superposition of radio sources seen in Stokes parameter $I$ on an EIT $17.1 \mathrm{~nm}$ image $(b)$ for the stationary type IV source on March 4, 2012. The contours of equal brightness temperature at $50 \%$ of the maximum are plotted in colours to represent different frequencies.

sense of polarisation and the photospheric magnetic polarity was not possible for this event.

The location near the limb offers the opportunity to locate the radio sources with respect to the CME. To this end we made a 3D reconstruction of the CME front by using a combination of coronagraphic images at 11:00 UT taken by LASCO C2 and the COR1 coronoagraph of the SECCHI suite (Howard et al. 2008) on board the STEREO B satellite, respectively, using the 3D creation software Blender ${ }^{5}$. Figure E.3b shows the 3D reconstruction of the CME front. The NRH contours seen in Stokes $V$ at four different frequencies are overplotted. The elongated contour contains the moving type IV source at its top and the southern stationary type IV source at its bottom. The northern radio source plotted by red contours is the persistent component of the stationary type IV continuum. The radio sources were located under the CME span, with the two components of the station-

\footnotetext{
5 https://www.blender.org/
}

b)
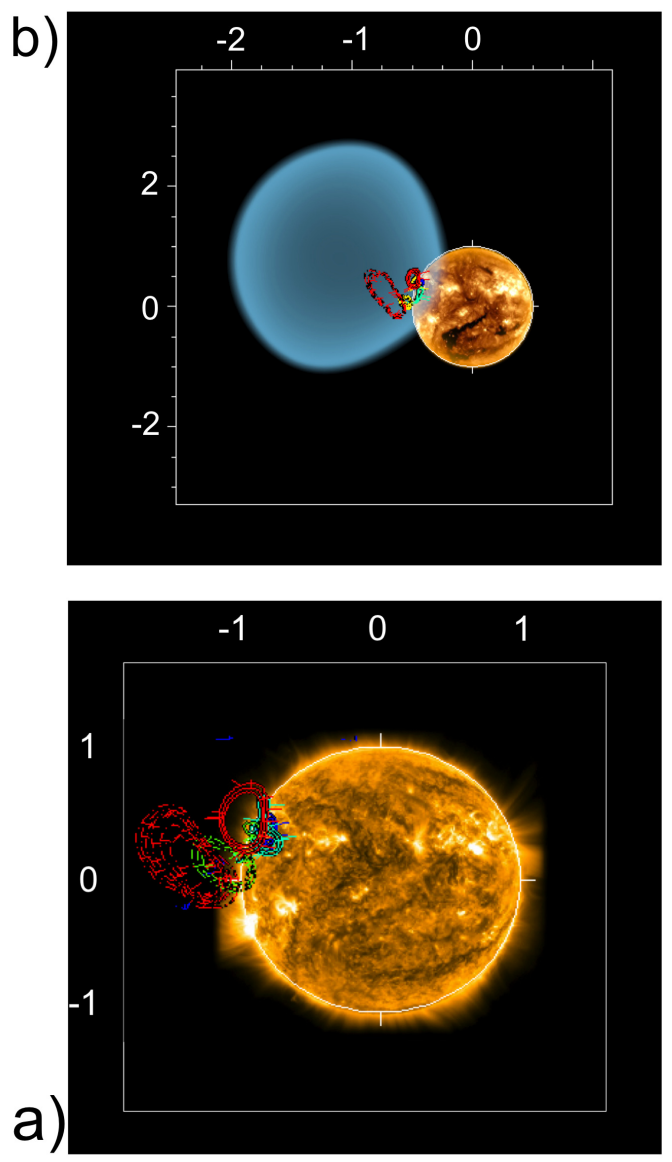

Fig. E.3. $a$ : superposition of radio sources seen in Stokes parameter $V$ on SDO AIA 171 image on March 4, 2012. $b$ : 3D visualisation of the CME front at 11:00 UT by using the Blender software, with overlaid radio sources in Stokes parameter $V$. In both images the iso-intensity contours at $50 \%$ of the maximum are plotted in colours to represent the different frequencies: blue for $432 \mathrm{MHz}$, green for $327 \mathrm{MHz}$, yellow for $228 \mathrm{MHz}$ and red for $150 \mathrm{MHz}$.

ary type IV burst near the flanks. The configuration is reminiscent of the CSHKP scenario (Carmichael 1964; Sturrock 1966; Hirayama 1974; Kopp \& Pneuman 1976), which displays a twodimensional cut of an erupting magnetic flux rope.

\section{Appendix F: June 14, 2012}

The event was associated with a halo CME with speed of about $1000 \mathrm{~km} \mathrm{~s}^{-1}$. It was first observed at 14:12 UT by LASCO C2. This Earth-directed CME was related to a flare in the active

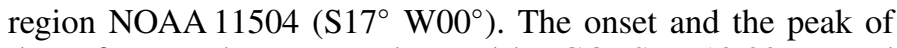
the soft X-ray burst were detected by GOES at 13:20 UT and 14:30 UT, respectively, as shown in Fig. F.1a. Flare loops were first observed between 13:44 UT and 13:52 UT in AIA $19.3 \mathrm{~nm}$ images. Figures F.1b and c present 1D images taken by NRH at $150 \mathrm{MHz}$ projected on the solar north-south and east-west directions, respectively. A noise storm was observed accompanied by sporadic sources before the soft X-ray burst onset, and followed by moving type IV sources between about 13:30 and 14:00 UT. They were analysed in detail by Morosan et al. (2020). The stationary type IV emission is the long lasting source observed at $150 \mathrm{MHz}$ (enclosed in blue rectangles) after 14:10 UT. The NRH observed this emission at all frequencies $(150-445 \mathrm{MHz})$. Its spectrum extended through the frequency range of the NDA, and 


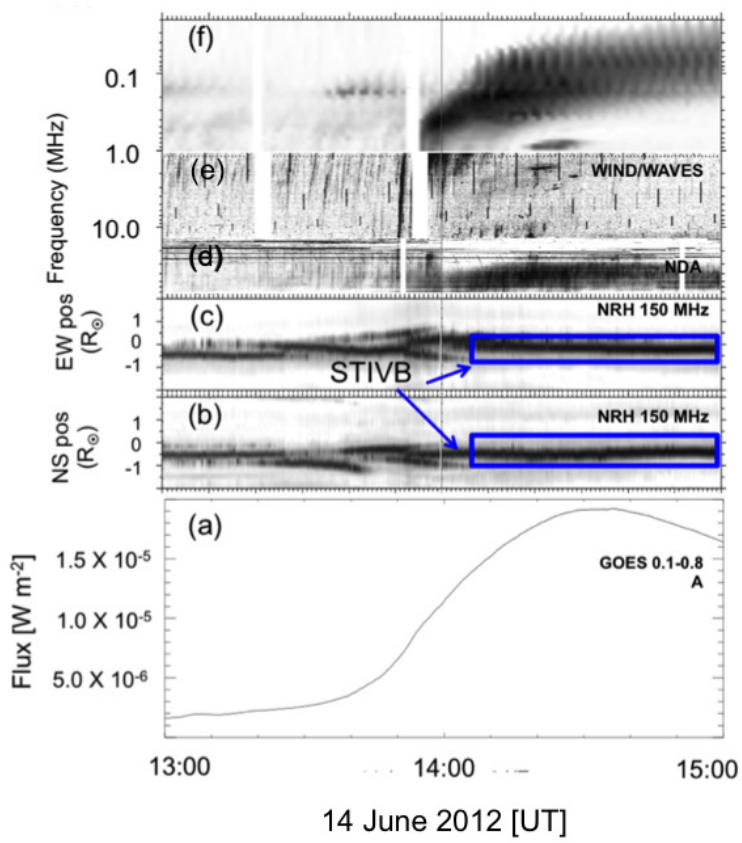

Fig. F.1. Multifrequency plot. Panels show: (a) soft X-ray profile; $(b)$ 1D images projected on the solar north-south and $(c)$ east-west directions ( $y$ axis graded in solar radii) at $150 \mathrm{MHz}$; the stationary type IV burst (STIVB) is enclosed by the blue rectangles. $(d)-(f)$ : dynamic spectra between $80 \mathrm{MHz}$ and $10 \mathrm{kHz}$.

traces were seen at the high-frequency border of Wind/WAVES. The apparent gap between the low-frequency part of the NDA spectrum and the Wind/WAVES spectrum is probably due to the reduced sensitivity of the NDA antennas. The spectrum of the stationary type IV burst hence extended down to $12 \mathrm{MHz}$.

\section{F.1. Geometry of the radio source and the magnetic field}

Figure F.2 shows the overlay of the source centroids observed by the NRH at $432 \mathrm{MHz}$ during the noise storm (b) and the stationary type IV continuum (c) on the photospheric magnetogram and its PFSS extrapolation. Different colours correspond to different times. The stationary type IV source in Fig. F.2c had two components. Both had columnar structure with opposite senses of polarisation. They were observed during most of the time until the end of NRH observations. The two columnar radio sources project onto the two legs of an extended loop structure in the PFSS extrapolation, which connect the leading and the following part of the underlying active region. The evolution of the brightness temperatures of the two sources, displayed in the bottom panel of Fig. F.3, is correlated. This confirms that the two columnar radio sources were indeed part of a common large-scale magnetic structure, which in the presence of coronal currents is a flux rope instead of a simple loop inferred from the PFSS extrapolation. Each of the two source locations project onto a dimming region observed in EUV images as shown in Fig. F.4a.

\section{F.2. Circular polarisation and the mode of radio emission}

The location of the left-hand polarised component $(V>0)$ coincided with the positive polarity region in the photospheric magnetic field, while the right-hand polarised radio source was located above the negative polarity region (Fig. F.2c). This implies that the bipolar source was emitting in the ordinary c)

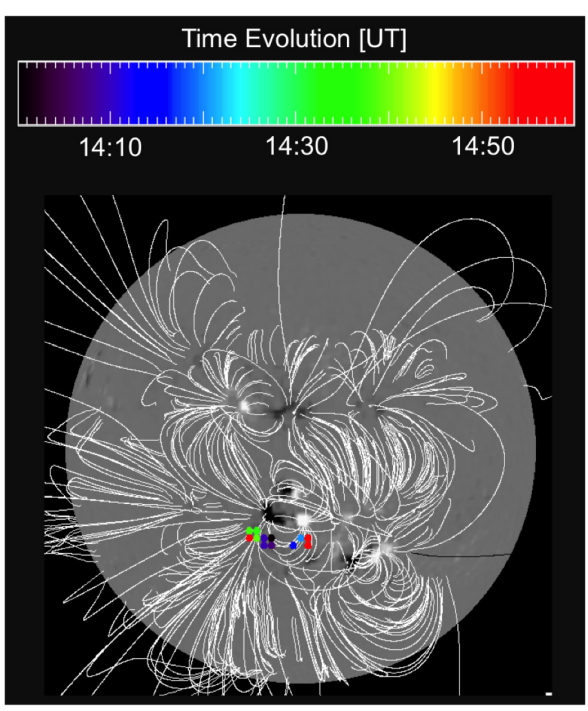

b)

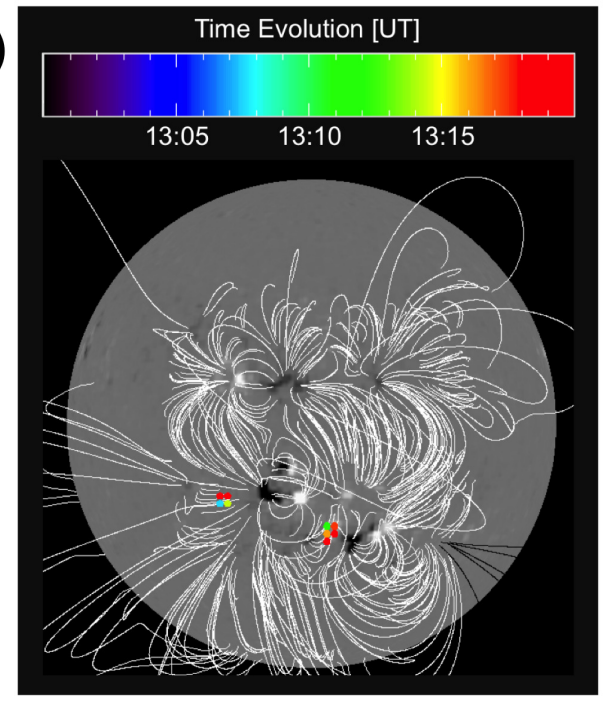

a)

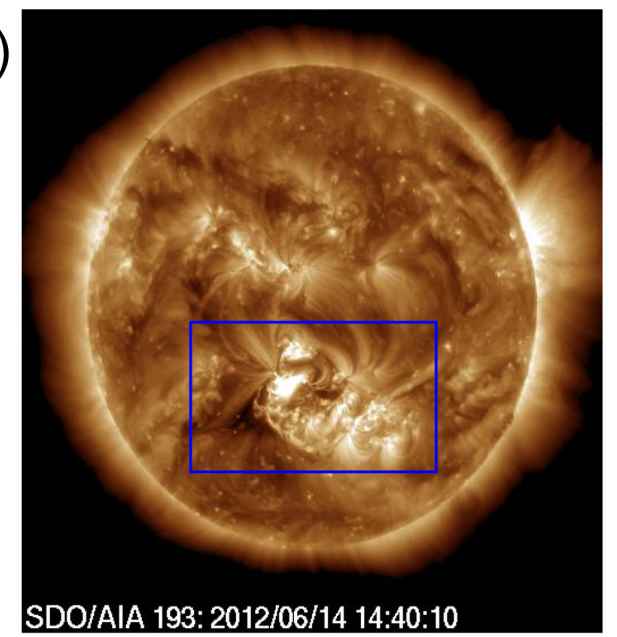

Fig. F.2. Localisation of the source centroids on June 14, 2012 at $432 \mathrm{MHz}$. $a$ : SDO AIA image showing the active region NOAA 11504 enclosed by the blue rectangle. $b, c$ : colour-coded time evolution overlaid on the magnetic field configuration inferred by the PFSS model for the noise storm $(b)$ and the stationary type IV continuum $(c)$.

mode. The time histories of the polarisation degrees are plotted in the top panel of Fig. F.3. While the brightness temperatures 

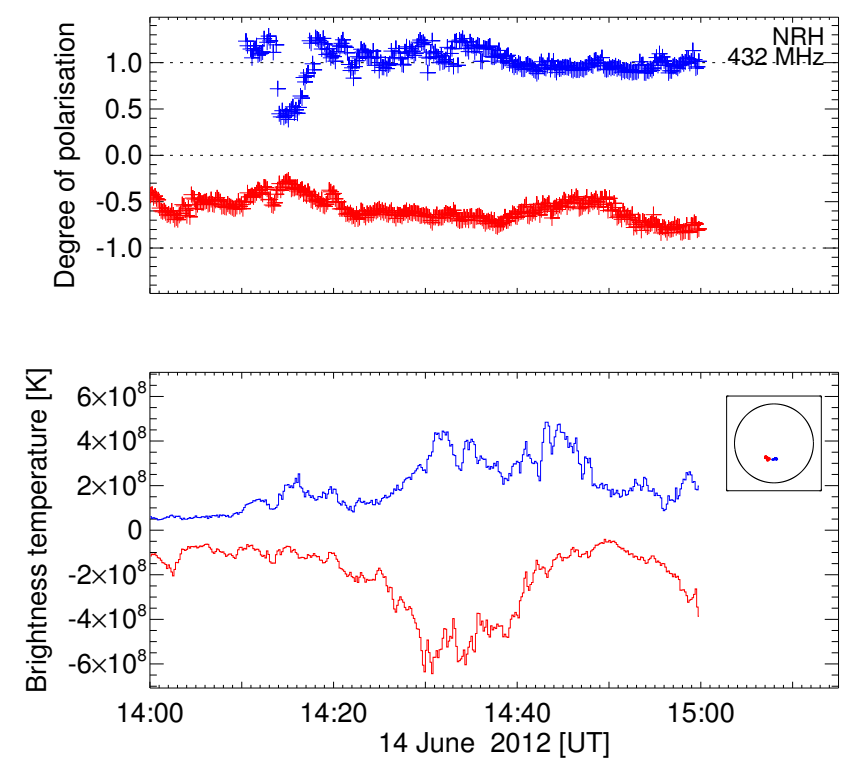

Fig. F.3. Top panel: polarisation profile of the two stationary type IV sources on June 14,2012 at $432 \mathrm{MHz}$ in the time range of 14:00 UT-15:00 UT. Bottom panel: brightness temperature profile of two radio sources. The blue and red curves refer to the source with $V>0$ and with $V<0$, respectively. Integration time $10 \mathrm{~s}$.

(bottom panel) varied, the degrees of circular polarisation were essentially constant. The average values of the degree of circular polarisation and the logarithm of the brightness temperature in Col. 8, 9 of Table 1 were computed in the interval 14:20-15:15 UT.

The western source is fully left-hand polarised, while the eastern one is partially right-hand polarised throughout. The partial polarisation is a solar effect, and not due to the antenna beam, since it is the brighter source that is partially polarised, while the weaker source is fully polarised. Several sources with different degrees of polarisation were occasionally observed in the eastern source, as is shown in Fig. F.4b. Black contours denote Stokes parameter $I$ while the red ones indicate Stokes parameter $V$. The figure shows that the polarised part occupies only a fraction of the area of the eastern source in total intensity. This shows that a part of the overall source is strongly polarised, while another part has weak or no polarisation. This observation is an important hint to the interpretation of partial polarisation by the mixture of substructure with strong and weak polarisation.

\section{F.3. Correlated evolution of brightness temperatures in the two sources}

A closer inspection of the brightness temperature profiles of the two oppositely polarised sources in Fig. F.3 reveals a delay of the western source. The cross-correlation of the curves yields a delay of about $36 \mathrm{~s}$. To explore if this delay can be explained by the travel of electrons along the magnetic flux rope, we analysed the STEREO B/COR1 images. The CME presented a cavity structure whose core appeared in the field of view of the coronagraph after 13:40 UT. Since the core has been identified as the flux rope in white-light images of CMEs (e.g. Howard et al. 2017; Song et al. 2019), we use its heliocentric distance, about $3 R_{\odot}$, to estimate the height of the flux rope. From the location of the two radio sources at high frequencies $(432 \mathrm{MHz})$ we infer that the two legs of the flux rope were distant by about $14^{\circ}$. The path length was roughly calculated using the height of the b)

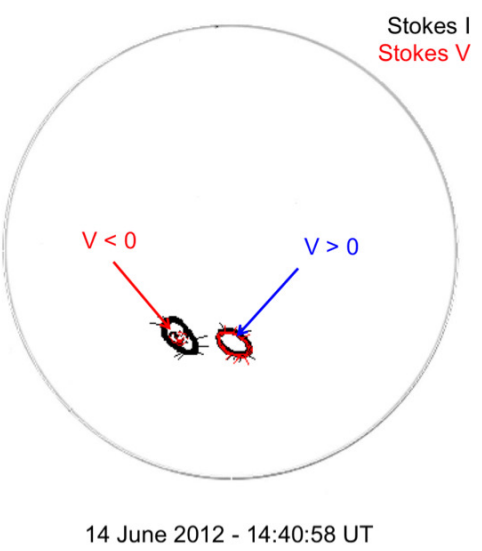

a)

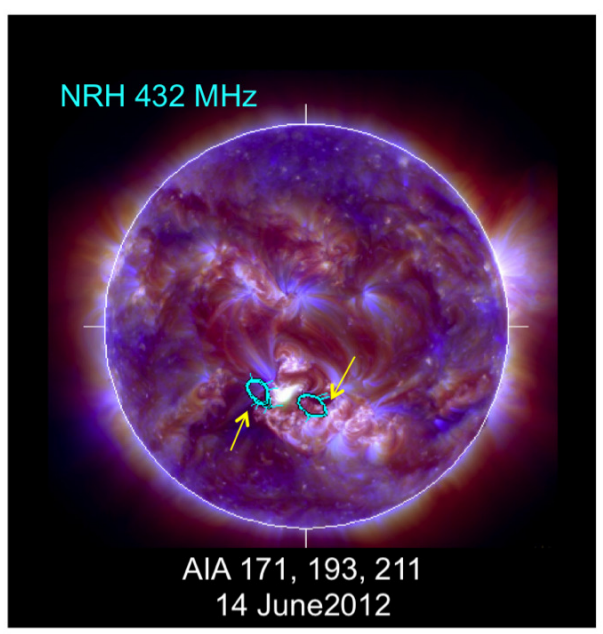

Fig. F.4. Radio sources at $432 \mathrm{MHz}$. $a$ : location of radio sources seen in Stokes parameter $I$. The contours of equal brightness temperature at $50 \%$ of the maximum are plotted on a combination of AIA images $(17.1,19.3$ and $21.1 \mathrm{~nm})$. The yellow arrows indicate the location of dimmings. $b$ : radio sources seen in Stokes $I$ (black) and $V$ (red).

CME core above the photosphere and the arc connecting them at the top. The overall length travelled by the electrons from one foot of this model flux rope to the other was found to be about $3.0 \times 10^{9} \mathrm{~km}$. Electrons travelling this distance in $36 \mathrm{~s}$ have a field-aligned speed of about $0.28 c$, and a kinetic energy of at least $20 \mathrm{keV}$.

\section{F.4. The noise storm}

As on July 14, 2000 and October 9, 2001, noise storms were identified and localised at two different places in MDI magnetograms in Fig. F.2b. The eastern noise storm was right-hand polarised. Its source location in Fig. F.2b is east of the large-scale magnetic structure hosting the stationary type IV sources. This noise storm source was located in large-scale loops that connect the trailing, negative polarity of the parent active region to its eastern surroundings. The western, left-hand polarised noise storm was connected to the leading part of the active region, with northward (positive) polarity. Both noise storms were strongly polarised, between $60 \%$ at the low frequencies and $80 \%$ at the highest frequency. The polarities of the two noise storms and the two stationary type IV sources are all consistent with radio emission in the ordinary mode. 